${ }^{\circledR}$ Entomologica Fennica. 8 May 2000

\title{
A synopsis of the jumping spider fauna in the Russian Far East (Araneae, Salticidae)
}

\author{
Dmitri V. Logunov \& Seppo Koponen
}

Logunov, D.V. \& Koponen, S. 2000: A synopsis of the jumping spider fauna in the Russian Far East (Araneae, Salticidae). — Entomol. Fennica 11: 67-87.

A revised check-list of the jumping spiders in the Russian Far East is given. Altogether, 82 valid species are listed on the basis of literature and examination of new materials. Besides, 15 species previously reported from the area are considered to be wrong or doubtful records. All literature records from the Russian Far East with original nomenclature are included, and the collection localities are shown on maps.

Dmitri V. Logunov, Siberian Zoological Museum, Institute for Systematics and Ecology of Animals, Siberian Division of the Russian Academy of Sciences, Frunze Street 11, Novosibirsk,630091, Russia.e-mail:dpavuk@online.nsk.su

Seppo Koponen, Zoological Museum, Centre for Biodiversity, University of Turku, FIN-20014 Turku, Finland. e-mail: sepkopo@utu.fi

Received 18 February 1999, accepted 25 January 2000

\section{Introduction}

The earliest records of the jumping spiders in the Russian Far East were by Grube (1861), who described 9 valid species from Cisamuria. Thereafter the Salticidae of the area at hand got only little attention (Kulczynski 1885, 1895, 1926, Schenkel 1930, Peelle \& Saito 1933, Sytshevskaja 1935) until the important publication of Prószynski (1979) on the Salticidae of the USSR was published. The latter work doubtless laid investigations of the salticids in Russia on a regular base and, as a result, the first faunistic review of the jumping spiders in the Russian Far East numbering 51 species was soon published (Dunin 1984).

Since then, a number of taxonomic and faunistic works on the Salticidae in the Russian Far
East have been published (Marusik 1988, Logunov \& Wesolowska 1992, 1995, Logunov et al. 1993, Logunov 1998a, 1998b, Logunov \& Marusik 1991, 1994, 1999b; etc.). Recent regional check-lists of spiders (e.g. Marusik et al. 1992, 1993) also included valuable information about the Salticidae. The present paper thus gives a full up-to-date account of the 82 salticid species recorded in the Russian Far East, including both original and literature data. Besides, 15 doubtful and/or wrong records are specially discussed.

\section{Material and methods}

The Russian Far East is accepted as the territory lying to the east of Yakutia (=Yakut Saha Republic) and the Chita Area 
(Fig. 1, A). Subdivision of the area and almost all studied localities are shown in Figs. 1-2. In the text, each locality is followed by the respective number in square brackets ([]) and referring to the locality number in Figs. 1-2. The few localities put in quotes («») refer to those we have been unable to find on available maps; in most cases their published spelling was wrong or/and they reflect old names, which have been changed during last decades. The section «Localities» under each species includes all the literature data, while all new area/province records are given in «Material examined». The up-to-date check-list of species is preceded by the list of wrong or/and doubtful records (15 altogether).

Only relevant literature concerning the spider fauna of the territory studied is included in the text. For a complete list of taxonomic sources for each species involved, see Prószynski (1990).

The studied or revised specimens are shared between the following museums:

FSCA - Florida State Collection of Arthropods FDACS, Division of Plant Industry, Gainesville, Florida, U.S.A;

ISEA - Institute for Systematics and Ecology of Animals, Novosibirsk, Russia;

SMNH - Swedish Museum of Natural History, Stockholm, Sweden;

ZISP - Zoological Institute, St-Petersburg, Russia;

ZMMU - Zoological Museum of the Moscow State University, Moscow, Russia;

ZMTU - Zoological Museum of the University of Turku, Turku, Finland.

The names of most collectors are abbreviated as follows: V.D. - V.V. Dubatolov; K.E. - K.Yu. Eskov; K.M. - K.G. Mikhailov; T.O. - T.I. Oliger; S.G. - S.I. Golovatch; Y.M. Yu.M. Marusik; S.K. - S. Koponen. Other abbreviations used are: Isl. - Island; Res. - Reservation.

\section{Wrong and/or doubtful records}

Chalcoscirtus micans (Caporiacco, 1935). C. micans was reported for Magadan Area by Marusik (1988), but later this species was shown (Marusik 1991 ) to be a junior synonym of $C$. glacialis.

Evarcha falcata (Clerck, 1758). All the former records of $E$. falcata from the Russian Far East (Kulczynski 1885, Sytshevskaja 1935, Azheganova \& Stenchenko 1977, Prószynski 1979, Dunin 1984, Logunov \& Wesolowska 1992, Marusik et al. 1992, Marusik et al. 1993, Kim \& Kurenshchikov 1995) in reality belong to $E$. proszynskii. For more details, see Marusik \& Logunov (1998).

Icius nigra Peelle \& Saito, 1933. This species was described from the Kurile Islands (Iturup) (Peelle \& Saito 1933) and since that time has not ever been reported. The type of this species, like the most Saito's types, seems to be lost and hence

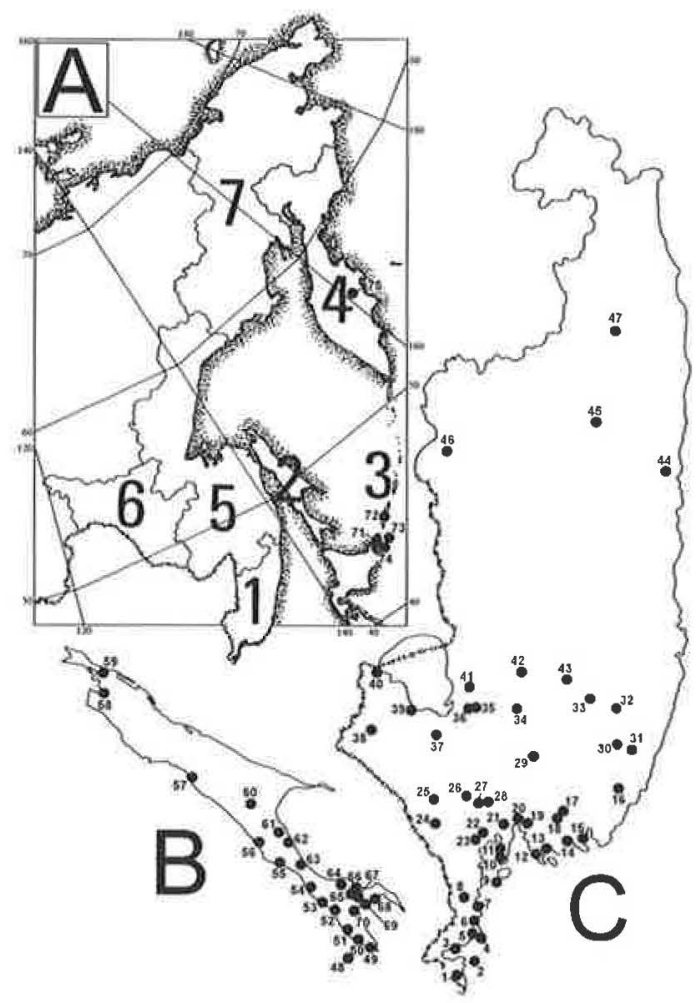

Fig. 1. Administrative subdivision of the Russian Far East (A) and collecting localities in Sakhalin (B), Maritime Province (C), Kurile Islands (3 in A) and Kamchatka (4 in A). - A: Administrative units 1. Maritime Province; 2. Sakhalin; 3. Kurile Islands; 4. Kamchatka; 5. Khabarovsk Province; 6. Amur Area; 7. Magadan Area. - B-C: Collecting localities C: Maritime Province: 1. Khasan; 2. Furugel'ma Isl.; 3. Pos'et Bay; 4. Gamova Peninsula; 5. Andreevka; 6. Ryazanovka; 7. Slavyanka; 8. Kedrovaya Pad' Res.; 9. Popova Isl.; 10. Vladivostok; 11. Sadgorod, 12. Tikhookeanskiy; 13. Domashlino; 14. Dushkino; 15. Nakhodka; 16.Tchistovodnoe; 17. Tigrovoi; 18. Anisimovka; 19. Smolyaninovo; 20. Shkotovo; 21. Artem; 22. Kiparisovo; 23. Vinevitino; 24. Monakino; 25. Pokrovka; 26. Ussuriysk; 27. Gornotaezhnoe; 28. Ussuriysky Res.; 29. Vinogradovka; 30. Lazo Town; 31. Lazo Res.; 32. Oblachnaya Mt.; 33. Pravaya Izvilinka River; 34. Chernyshevka; 35. Merkushevka; 36. Dmitrievka; 37. Khorol'; 38. Barabash-Levada; 39. Kamen'-Aybolov; 40. Turiy Rog; 41. Spassk-Dal'niy; 42. Yakovlevka; 43. Chuguevka; 44. Sikhote-Alin' Res.; 45. Blagodatnyi; 46. middle reaches of Bikin River; 47. Ulunga River. - B: Sakhalin: 48. Moneron Isl.; 49. Kril'on Peninsula; 50. Kuznetsovo; 51. Nevel'sk; 52. Kholmsk; 53. Kostromskoe; 54. Tomari; 55. Ainskoe Lake; 56. Uglegorsk; 57. Alexandrovsk-Sakhalinsk; 58. Beryozovka River; 59. Okha; 60. Smirnykh; 61. Nitui River; 62. Makarov; 63. Pugatchevo; 64. Dolinsk; 65. Novo-Alexandrovsk; 66. Chekhov's Peak; 67. Yuzhno-Sakhalinsk; 68. Ozerskiy; 69. Korsakov; 70. Aniva. - A: Kurile Islands: 71. Kunashir Isl.; 72. Iturup Isl.; 73. Shikotan Isl.; 74. Zelyonyi Isl. - A: Kamchatka: 75. Klyuchevskoe. 


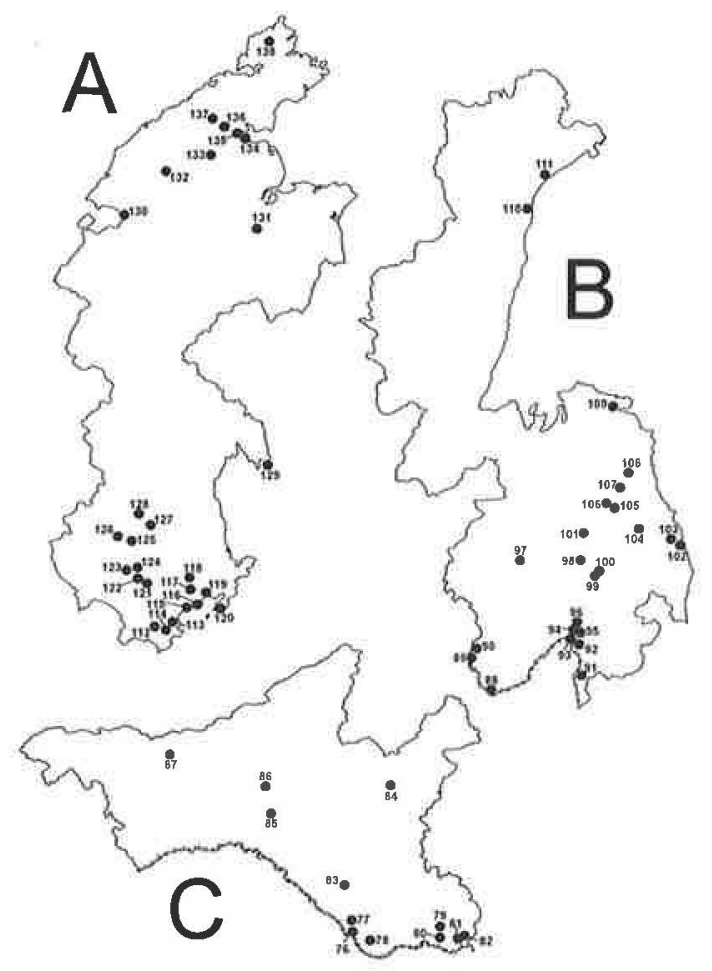

Fig. 2. Collecting localities in Magadan Area (A), Khabarovsk Province (B) and Amur Area (C). - C: Amur Area: 76. Blagoveshchensk; 77. Belogorie; 78. Sadovoe; 79. Arkhara; 80. Lebedinskoe Lake; 81. Khingan Res.; 82. Kundur; 83. Malaya Sezanka; 84. Selemdzhinsk; 85. Ulunga River; 86. Zeisky Res.; 87. Tyndinskiy. - B: Khabarovsk Province: 88. ca. 409 km of Khabarovsk; 89. Pashkovo; 90. Obluchie; 91. Boitsovo; 92. Kiya River; 93. Bolsjoi Khetsyr Mt. Range; 94. Priamurskaya Station; 95. Korfovskiy; 96. Kabarovsk, Zelyonyi Isl. and Rybachiy Isl.; 97. Mogdy River, Badzhal Mts; 98. Bolon' Lake; 99. Troitskoe; 100. Slavyanka; 101. Komsomol'skna-Amure; 102. Vanino; 103. Akur River; 104. Vysokogornyi; 105. Uktur River; 106. Nizhnetambovskoe; 107. Tsymmermanovka; 108. Sofiysk; 109. Nikolaevskna-Amure; 110. Ulia River; 111. Okhotsk. - A: Magadan Area: 112 . the middle reaches of Cholomdzha River; 113. Talon Town; 114. Kava River; 115. Arman' Pass; 116. Magadan, Dukcha River; 117. Yablonevyi; 118. the upper reaches of Ola River, Talaya River mouth; 119. Gadlya; 120. Koni Peninsula; 121. Ust'-Omtchug, Detrin River basin; 122. Vakkhanka River mouth; 123. Sibit Tyellakh River basin, Kyunebellakh spring; 124. Vetrennyi; 125. Kulu, Kontakt Field Station; 126. Shirokiy; 127. Ust'Srednekan; 128. Seimtchan Town; 129. Taigonos Peninsula; 130. Chaun Bay; 131. Anadyr' River; 132. Bol'shaya Osinovaya River, 133 - Vul'vyveem River, 134 - Kresta Bay; 135. Egvekinot; 136. Amguema River; 137. 164 km Egvekinot-lul'tin; 138. the middle reaches of Chegitun' River. cannot be re-examined. However, even from the original figures of Peelle \& Saito (1933), it is evident that the species does not belong to Icius but seems to be a member of Phintella. The validity of Icius nigra needs a further study and confirmation.

Marpissa pichoni (Schenkel, 1963). This species was once reported from the Maritime Province by Dunin (1984). However, this specific name is known (for details, see Logunov 1999) to be one of the junior synonyms of Mendoza canestrinii.

Marpissa radiata (Grube, 1859). This species was once reported from Kamchatka by Sytshevskaja (1935). However, this record is to be confirmed by the pertinent material, as it seemed to belong to $M$. pomatia. $M$. radiata is known (Logunov 1996) to spread eastward approximately to the Lake Baikal only.

Mendoza pulchra Prószynski, 1976. The record of Marpissa pulchra from the Khabarovsk Province, Pashkovo (Kim \& Kurenshchikov 1995) is in need of confirmation. According to Logunov (1999), M. pulchra seems to be a junior synonym of Mendoza nobilis, while all the former records of Marpissa pulchra from the Russian Far East (e.g. Logunov \& Wesolowska 1992) turned out to belong to either Mendoza nobilis or M. elongata.

Myrmarachne japonica (Karsch, 1879). This species was once reported from the Maritime Province (Lazo Res.) by Oliger (1984). However, from the published figures (op. cit.: figs. 1-2), it is clear that the author dealt with $M$. lugubris, as Yaginuma (1986: fig. 134.1) did as well. $M$. japonica, as shown by Prószynski (1973), was described from subadult males and their conspecifity with all the specimens recently reported/redescribed under this name (e.g. Oliger 1984, Song 1987) needs a careful revision. Another matter to be revised in the future is a possible conspecifity of $M$. japonica and $M$. lugubris.

Myrmarachne inermichelis Bösenberg \& Strand, 1906. M. lugubris was erroneously reported by Oliger (1984) from the Russian Far East (Lazo Res.) under the name $M$. inermichelis (Oliger's specimens re-examined).The latter species has so far been reported from Japan and Taiwan only (Prószynski 1990). 
Phintella abnormis (Bösenberg \& Strand, 1906). All the records of this species from the Maritime Province (Prószynski 1979, Dunin 1984) turned out to belong to P. popovi (see Logunov \& Wesolowska 1992).

Pellenes (s.str.) tripunctatus (Walckenaer, 1802). All the Far Eastern records of $P$. tripunctatus (Kulczynski 1926, Sytshevskaja 1935, Marusik 1988, Marusik et al. 1992) turned out to refer to $P$. sibiricus. For more details, see Logunov \& Marusik (1994).

Pseudeuophrys erratica (Walckenaer, 1826). All the former records of Euophrys erratica from the Russian Far East (Prószynski 1979) in reality turned out to refer to Pseudeuophrys iwatensis (see also Logunov 1998b). However, the occurrence of $P$. erratica in the Russian Far East is possible, as it was with certainty recorded from Japan, Hokkaido (Matsuda 1991, Ikeda 1996, both sub Euophrys erratica).

Sitticus avocator (O.P.-Cambridge, 1885). This species has been reported from the Maritime Province of the Russian Far East (Prószynski 1979: sub S. viduus, Dunin 1984, Logunov 1998a), as well as from Korea and Japan (see Prószynski 1990 for a complete set of references). However, all the former records of $S$. avocator (=viduus) from the Far East have to be referred to $S$. distinguendus (Logunov, personal data). The problem will be considered in details separately.

Sitticus caricis (Westring, 1861). This species has so far been reported three times from the Russian Far East: Khabarovsk Prov., Liman of Amur (=Nikolaevsk-na-Amure) (Prószynski 1979); Sakhalin, Okha (Marusik et al. 1993) and Kamchatka (Kulczynski 1885). Unfortunately, we have been unable to revise $S$. caricis specimens of the above authors, and so it is uncertain if the species does occur in the Far East. The problem calls for a special attention in the future.

Sitticus pubescens (Fabricius, 1775). This European species was first reported from Cisamuria by Grube (1861) and since that the record has been repeated by Charitonov (1932), Azheganova \& Stenchenko (1977), Dunin (1984) and Nenilin (1985). However, $S$. pubescens was found to be absent from Grube's collection deposited in the Wroclaw Zoological Museum (see Prószynski 1971), therefore it is unclear which species was identified by Grube (1861) under this name.
Sitticus terebratus (Clerck, 1758). Kulczynski (1895) reported a single female from the Ussuri region. This record needs confirmation from pertinent material. According to Dunin's figures (Dunin 1984: figs 61, 62), this author reported either a new species, or Harmochirus latens under the name $S$. terebratus. The problem is open until re-examination of Dunin's specimens (unknown museum depository).

\section{Revised check-list of species}

\section{Aelurillus v-insignitus (Clerck, 1758)}

Aelurillus v-insignitus: Marusik 1988: 1482; Marusik et al., 1992: 151.

Localities. Magadan Area: Sibit-Tyellakh River basin [123] (Marusik 1988), Kava River [114], Kulu [125], Vetrennyi [124], Ust'-Omtchug [121], Yablonevyi [117] (Marusik et al. 1992).

\section{Asianellus festivus (C.L. Koch, 1834)}

Attus melanotarsus: Grube 1861: 24.

Aelurillus festivus: Prószynski 1971: 209-210, figs 7-9; Ibid., 1979: 302; Dunin 1984: 128-129, fig.1; Sternbergs 1988: 93; Logunov \& Wesolowska 1992: 115; Marusik et al. 1992: 151; Kim \& Kurenshchikov 1995: 64 .

Asianellus festivus: Logunov \& Heciak 1996: 106108, figs 1-5, 8, 10, 17-19, 23-28, 35, 39.

Localities. Khabarovsk Prov.: Okhotsk [111] (Prószynski 1979), Ulia River [110] (Marusik et al. 1992), Bolshoi Khekhtsyr Mt. Range [93] (Logunov \& Wesolowska 1992), Komsomol'sk-naAmure [101], «Voronezhsky Hills», Priamurskaya Station [94], Slavyanka [100] (Kim \& Kurenshchikov 1995). - Maritime Prov.: Ryazanovka [6], Furugel'ma Isl. [2], Pos'et Bay [3] (Sternbergs 1988, Logunov \& Heciak 1996), Khanka [39], Vladivostok [10], Kangauz (=Anisimovka) [18] (Prószynski 1979, Dunin 1984).

Material examined. 10 (FSCA), Amur Area, near Blagoveshchensk, Peschanoe Lake [76], 12.06.1996, E.I.Malikova; 3 ơ $^{*}$ (ISEA), same area, near Blagoveshchensk, 7-8 km NE of Mokhovaya Pad', Peschanoe Lake [76], 4-5.07.1999, V.D.; $7 \sigma^{\pi} \sigma^{\pi}, 3$ 우 우 (ISEA), same area, near Blagoveshchensk, Verkhneblagoveshchenskoe [76], 14.05.4.08.1999, V.D.; 4 ơ (ISEA), Maritime Prov., ca. $5 \mathrm{~km} \mathrm{~S} \mathrm{of}$ Smolyaninovo, Romanovka [19], 1-13.05.1994, V.D.; $1 \sigma^{*}$ 
(ISEA), same area, ca. $20 \mathrm{~km}$ SE of Ussuriysk, Gomotaezhnoe [27], 1-2.06.1994, V.D.; $1 \sigma^{\star}, 1$ ㅇ (ISEA), same area, Gamova Peninsular, Vityaz' Bay [4], 20-23.05.1994, V.D.; $5 \sigma^{\circ} \sigma^{*}$, 4 + (ISEA), $30^{*} o^{*}$ (ZMMU), same area, Lazo Res., $43^{\circ} 16^{\prime} \mathrm{N}$, $134^{\circ} 08^{\prime} \mathrm{E}$ [31], 28.08.1975-20.09.1981, T.O.; 1 \% (ZMTU), same area, SE part, ca. $25 \mathrm{~km} \mathrm{NE}$ of Nakhodka [15], 18.08.1998, Y.M.; $10^{*}$ (ZMTU), same area, S part, Pravaya Izvilinka River, 4352'N, 134'17'E [33], 2.08.1998, Y.M.; $10^{*}, 2$ 우 (ISEA), Kurile Islands, Kunashir Isl., ca. 2 km N of Krugly Cape, $140^{\circ} 41^{\prime} \mathrm{E}, 44^{\circ} 01^{\prime} \mathrm{N}$ [71], 2-23.09.1997, Y.M.

\section{'Bianor” aemulus (Gertsch, 1934)}

Bianor aurocinctus (misidentification): Marusik 1988: 1482.

Bianor aemulus: Logunov \& Marusik 1991: 4146, figs 1d-k, 2a-d,k, 4; Marusik et al. 1992: 151.

Localities. Magadan Area: Sibit-Tyellakh River basin [123] (Marusik 1988: sub Bianor aurocinctus, Logunov \& Marusik 1991), Vetrennyi [124], Kontakt Field Station [125], Talon Town [113] (Marusik et al. 1992).

\section{“'Bianor"'aurocinctus (Ohlert, 1865)}

Bianor aenescens: Dunin 1984: 130.

Bianor aurocinctus: Logunov \& Marusik 1991: 39-40, figs 1a-b, 2a-b, 3; Marusik et al. 1993: 82; Kim \& Kurenshchikov 1995: 64.

Localities. Khabarovsk Prov.: Slavyanka [100], Boitsovo [91], Bolshoi Khekhtsyr Mt. Range [93] (Kim \& Kurenshchikov 1995). Maritime Prov.: Vladivostok [10] (Dunin 1984). - Sakhalin: Chekhov Peak [66], Novo-Alexandrovsk [65], Korsakov [69] (Logunov \& Marusik 1991), Aniva [70], Okha [59], Smirnykh [60] (Marusik et al. 1993).

Notes. The records from Vladivostok (Dunin 1984) and Khabarovsk Province (Kim \& Kurenshchikov 1995) are doubtful, they may turn out to belong to "Harmochirus" nigriculus (see below).

\section{Carrhotus xanthogramma (Latreille, 1819)}

Carrhotus xanthogramma: Prószynski 1979: 304, fig. 17; Dunin 1984: 130, figs 3, 4; Logunov \& Wesolowska 1992: 115; Marusik et al. 1993: 82; Kim \& Kurenshchikov 1995: 64.

Carrhotus detritus: Sternbergs 1988: 93.
Localities. Khabarovsk Prov.: «Malvinskoe» (?) near Okhotsk sea (Prószynski 1979), Korfovsky [95] (Dunin 1984), Bolshoi Khekhtsyr Mt. Range [93], Slavyanka [100] (Logunov \& Wesolowska 1992), Pashkovo [89], Priamurskaya Station [94] (Kim \& Kurenshchikov 1995). - Maritime Prov.: Vladivostok [10], Monakino [24], Tikhookeanskiy [12], Blagodatnyi [45], Kedrovaya Pad' Res. [8], Barabash-Levada [38] (Dunin 1984), Furugel'ma Isl. [2] (Sternbergs 1988). — Kurile Islands: Kunashir [71] (Marusik et al. 1993).

Material examined. 6 우 (ISEA), Amur Area, near Blagoveshchensk, Verkhneblagoveshchenskoe [76], 14.05.4.08.1999, V.D.; 1 o (ISEA), same area, south of Svobodnyi Town, Malaya Sezanka [83], 05.1999, V.D.; $3 \sigma^{\circ} \sigma^{*}$ (ISEA), Maritime Prov., Vladivostok [10], 19-28.05.1994, V.D.; 1 우 (ZMTU), same locality, near Anisimovka (=Kangauz) [18], 25.07.1998, S.K.; $10^{\star}$ (ISEA), same area, ca. $5 \mathrm{~km}$ S of Smolyaninovo, Romanovka [19], 1-13.05.1994, V.D.; $1 o^{\star}, 1$ ㅇ (ISEA), same area, ca. $20 \mathrm{~km}$ SE of Ussuriysk, Gornotaezhnoe [27], 1-2.06.1994, V.D.; $90^{\star} \sigma^{\star}, 8 \% 9$ (ISEA), $10^{\star}, 2$ 우 우

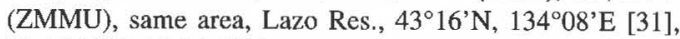
20.05.1975-15.05.1982, T.O.; $10^{*}, 1$ 우 (FSCA), same area, Anuchino Distr., 2-3 km N of Chernyshevka [34], 34.06.1994, V.D.; 10 (ISEA), same area, "Kedrovaya Pad"” Res. [8], 12.07.1976, B.P. Zakharov; 1 ㅇ (ZMMU), same area, near Ussuriysk, Kamenushka [26], 10.06.1989, S.Kurbatov; 19 (ZMTU), same area, W of Khanka Lake, near BarabashLevada, Cape, $44^{\circ} 46^{\prime} \mathrm{N}, 131^{\circ} 27^{\prime} \mathrm{E}$ [38], 18-19.07.1998, Y.M.; $2 \sigma^{*}$ (ZMMU), Khabarovsk Province, right bank of Amur River, ca. $20 \mathrm{~km} \mathrm{~N}$ of Troitskoye, Slavyanka [100], 1419.06.1990, S.G.

Chalcoscirtus (Chalcosibiricus) alpicola (L. Koch, 1876)

Chalcoscirtus alpicola: Marusik 1988: 1482; 1991: 21, fig. 1; Marusik et al. 1992: 151.

Localities. Magadan Area: Sibit-Tyellakh River basin [123], Kresta Bay [134], Vul'vyveem River [133], «Yanranaigytgyn Lake» (Marusik 1988, 1991, Marusik et al. 1992).

\section{Chalcoscirtus (s.str.) carbonarius Emerton, 1917}

Chalcoscirtus carbonarius: Marusik 1988: 1482; Marusik 1990: 53, fig. 3; Marusik et al. 1992: 151.

Localities. Magadan Area: Sibit-Tyellakh River basin [123], Kyunebellakh spring [123] (Marusik 1988, 1990). 


\section{Chalcoscirtus (Chalcosibiricus) glacialis Capo- racco, 1935}

Chalcoscirtus micans: Marusik 1988: 1482.

Chalcoscirtus glacialis: Marusik 1991: 22-24, ff. 2.1-2, 3.1-2; Logunov \& Marusik 1999a: 225. Chalcoscirtus glacialis sibiricus Marusik 1991: 24, figs 2.3-5, 3.3-4; Marusik et al. 1992: 151.

Localities. Magadan Area: Sibit-Tyellakh River basin [123], (Marusik 1988: sub Chalcoscirtus micans, Marusik 1991, Logunov \& Marusik 1999), Vetrennyi [124], Ust'-Omtchug [121], Ust'-Srednekan [127], the upper reaches of Bol'shay Osinovaya River [132] (Marusik et al. 1992), middle reaches of Anadyr' River [131] (Logunov \& Marusik 1999a).

\section{Chalcoscirtus (s.str.) grishkanae Marusik, 1988}

Chalcoscirtus grishkanae Marusik, 1988: 1475, fig. 4.

Chalcoscirtus grishkanae: Marusik 1991: 30; Marusik et al. 1992: 151.

Localities. Magadan Area: Sibit-Tyellakh River basin [123], Kontakt Field Station [125], Magadan [116] (Marusik 1988, 1991, Marusik et al. 1992).

\section{Chalcoscirtus (Chalcosibiricus) hype-boreus Marusik, 1991}

Chalcoscirtus hyperboreus Marusik, 1991: 25-26, figs 2, 3 .

Chalcoscirtus hyperboreus: Marusik et al. 1992: 151.

Localities. Magadan Area: Sibit-Tyellakh River basin [123], Kontakt Field Station [125], Vul'vyveem River [133], «Yanranaigytyn Lake», $164^{\text {th }} \mathrm{km}$ of highway Egvekinot-Iul'tin [137] (Marusik 1991), Egvekinot [135], the middle reaches of Chegitun' River [138] (Marusik et al. 1992).

\section{Chalcoscirtus (Chalcosibiricus) tanyae Logunov \& Marusik, 1999}

Chalcoscirtus tanyae Logunov \& Marusik, 1999b: 23-25, figs 1-4, 16.

Localities. Maritime Prov.: Anisimovka (= Kangauz) [18], Oblachnaya Mt. [32] (Logunov \& Marusik 1999b).

\section{Dendryphantes biankii Prószynski, 1979}

Dendryphantes biankii: Logunov \& Marusik 1994: 103-106, fig. 2.

Localities. Amur Area: Zeiski Res. [86] (Logunov \& Marusik 1994). — Khabarovsk Prov.: Bolshoi Khekhtsyr Mt. Range [93] (Logunov \& Marusik 1994).

\section{Dendryphantes czekanowskii Prószynski, 1979}

Dendryphantes czekanowskii: Marusik 1988: 1482; Marusik \& Cutler 1989: 51-53, figs 1-3; Marusik et al. 1992: 151; Kim \& Kurenshchikov 1995: 64.

Localities. Magadan Area: Sibit-Tyellakh River basin [123], Kontakt Field Station [125] (Marusik 1988, Marusik \& Cutler 1989), Bol'shaya Osinovaya River [132] (Marusik et al. 1992). Khabarovsk Prov.: Badzhal Mts [97] (Kim \& Kurenshchikov 1995).

\section{Dendryphantes fusconotatus (Grube, 1861)}

Attus fusconotatus Grube, 1861: 29.

Dendryphantes fusconotatus: Prószynski 1971: 210-211, figs 10-12; Ibid., 1979: 305-306, figs 37-63; Dunin 1984: 130, fig. 5; Marusik 1988: 1482; Logunov \& Wesolowska, 1992: 115; Marusik et al. 1992: 151; Marusik et al. 1993: 82; Kim \& Kurenshchikov 1995: 64.

Localities. Magadan Area: Sibit-Tyellakh River basin [123] (Marusik 1988), Shirokiy [126], Vetrennyi [124], Vakkhanka River mouth [122], the middle reaches of Cholomdzha River [112] (Marusik et al. 1992). - Khabarovsk Prov.: «Getcha», «Ozerpakh» (Prószynski 1979), «Kuznetsovsk» (Dunin 1984), Bolshoi Khekhtsyr Mt. Range [93] (Logunov \& Wesolowska 1992). - Maritime Prov.: Anisimovka (=Kangauz) [18], «Dzhanga», Vinogradovka [29] (Prószynski 1979). — Sakhalin: Okha [59] (Marusik et al. 1993).

Material examined. 1 (ISEA), Maritime Prov., Lazo Res., Korpad' Stand, $43^{\circ} 16^{\prime} \mathrm{N}, 134^{\circ} 08^{\prime} \mathrm{E}$ [31], 1.06.1980, T.O.; $4 \sigma^{\star} \sigma^{\star}, 1$ 우 (ISEA), same area, ca. $5 \mathrm{~km} \mathrm{~S}$ of Smolyaninovo, Romanovka [19], 1-13.05.1994, V.D. 


\section{Dendryphantes rudis (Sundevall, 1833)}

Dendryphantes rudis: Marusik 1988: 1482; Marusik et al. 1992; Marusik et al. 1993: 82.

Localities. Magadan Area: Sibit-Tyellakh River basin [123] (Marusik 1988, Marusik et al. 1992). - Sakhalin: Okha [59] (Marusik et al. 1993).

Material examined. 19 (ISEA), Amur Area, near Blagoveshchensk [76], summer 1996, A. Streltsov; 10 (ISEA), same area, near Blagoveshchensk, Verkhneblagoveshchenskoe [76], 14.05.-4.08.1999, V.D.

Notes. Schenkel's record of Denryphantes rudis from Kamchatka (Schenkel 1930) cannot be taken into consideration, because it was a juvenile specimen (see op. cit.: 4).

\section{Euophrys flavoatra (Grube, 1861)}

Attus flavoater Grube, 1861: 27.

Euophrys flavoatra: Prószynski 1971: 211-213, figs 13-15; Marusik et al. 1992: 151.

Euophrys flavoater: Logunov et al. 1993: 108111, figs 5-9; Kim \& Kurenshchikov 1995: 65.

Localities. Magadan Area: Kontaktovy Spring [125] (Logunov et al. 1993), the upper Kolyma [123] (Marusik et al. 1992). — Khabarovsk Prov.: Bolshoi Khekhtsyr Mt. Range [93], Nikolaevskna-Amure [109], Mogdy River [97] (Grube 1861, Logunov \& Wesolowska 1992, Logunov et al. 1993), Komsomol'sk-na-Amure [101], Badzhal Mts [97] (Kim \& Kurenshchikov 1995).

Material examined. 1 \& (ISEA), Maritime Prov., S part, Pravaya Izvilinka River, $43^{\circ} 55^{\prime} \mathrm{N}, 134^{\circ} 23^{\prime} \mathrm{E}$ [33], 12.08.1998, Y.M.

\section{Euophrys frontalis (Walckenaer, 1802)}

Euophrys frontalis: Dunin 1984: 130, figs 6, 7; Logunov et al. 1993: 111-113, figs 5, 10, 11; Marusik et al. 1993: 82.

Localities. Khabarovsk Prov.: «Gyrbykan», Bolshoi Khekhtsyr Mt. Range [93], Komsomol'sk-na-Amure [101], Mogdy River [97] (Logunov \& Wesolowska 1992, Logunov et al. 1993). - Maritime Prov.: Tikhookeanskiy [12], Artem [21] (Dunin 1984). — Sakhalin: Novo-Alexandrovsk [65] (Logunov et al. 1993), Okha [59] (Marusik et al. 1993).

Notes. All the records from the Maritime Province (Dunin 1984) need confirmation upon the pertinent material, they may turn out to be those of Euophrys kataokai.

\section{Euophrys kataokai Ikeda, 1996}

Euophrys frontalis (misidentification): Prószynski 1979: 306, figs 71-74.

Euophrys sp.: Logunov et al. 1993: 118-119.

Localities. Khabarovsk Prov.: Amur River at $409 \mathrm{~km}$ of Khabarovsk [88] (Logunov et al. 1993). - Maritime Prov.: Kedrovka River [8], Ussuriysky (=Suputinsky) Res. [28], «Teishgla» (Prószynski 1979: sub Euophrys frontalis).

Material examined. 1 \& (ISEA), Maritime Prov., SikhoteAlin' Res. [44], 29.05.1985, T.O.; 1 \%", 1 9 (ISEA), same area, Lazo Res., $43^{\circ} 16^{\prime} \mathrm{N}, 134^{\circ} 08^{\prime} \mathrm{E}$ [31], 15.06.1982, T.O.; 4 ㅇ

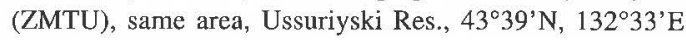
[28], 29-31.07.1998, Y.M.

\section{Euophrys proszynskii Logunov, Cutler \& Ma- rusik, 1993}

Euophrys frontalis: Marusik 1988: 1482.

Euophrys cf. frontalis: Marusik et al. 1992: 151. Euophrys proszynskii Logunov et al. 1993: 113117, figs 5, 12, 13.

Localities. Magadan Area: Sibit-Tyellakh River basin [123], Kontakt Field Station [125], Kyunnebellakh River Basin [123], Vakkhanka River [122], the upper reaches of Ola River [118] (Marusik 1988: sub Euophrys frontalis, Logunov et al. 1993), Arman' Pass [115], Gadlya [119], Ust'Omtchug [121], Vetrennyi [124], Ust'-Srednekan [127] (Marusik et al. 1992: sub Euophrys cf. frontalis).

\section{Evarcha albaria (L. Koch, 1878)}

Ergane albifrons Kulczynski 1895: 90-96, figs 2527.

Evarcha albaria: Prószynski 1979: 307, figs 8486; Dunin 1984: 131-132, figs 8-10; Logunov \& Wesolowska 1992: 115; Marusik et al. 1993: 82; Kim \& Kurenshchikov 1995: 65.

Localities. Khabarovsk Prov.: Bolshoi Khekhtsyr Mt. Range [93] (Logunov \& Wesolowska 1992), Komsomol'sk-na-Amure [101], «Voronezhsky Hills», Priamurskaya Station [94] (Kim \& Kurenshchikov 1995). - Maritime Prov.: Kedrovaya Pad' Res. [8], Ussuriysky (=Suputinsky) 
Res. [28], Anisimovka (=Kangauz) [18], Khanka Lake [39] (Prószynski 1979), Domashlino [13], Tikhookeanskiy [12], Blagodatnyi [45], Vladivostok [10], Barabash-Levada [38], Turiy Rog [40] (Dunin 1984). -- Kurile Islands: Kunashir [71] (Marusik et al. 1993).

Material examined. $1 \sigma^{*}$ (ISEA), Maritime Prov., Popova Isl. [9], 31.07.1978, B. P. Zakharov; 1 đ̛ (ISEA), same area, ca. $20 \mathrm{~km} \mathrm{SE}$ of Ussuriysk, Gornotayezhnoe [27], 12.06.1994, V.D.; $180^{*} \sigma^{*}, 20$ 우 (ISEA), $30^{\circ} 0^{*}, 4$ 우 (ZMMU), same area, S part, Lazo Res., $43^{\circ} 16^{\prime} \mathrm{N}, 134^{\circ} 08^{\prime} \mathrm{E}$ [31], 23.05.1978-22.06.1981, T.O.; $4 \sigma^{\star} \sigma^{\star}$ (ZMTU), $1 \sigma^{*}$ (ISEA) same locality, 6-9.08.1998, Y.M. \& S.K.; $2 \sigma^{*}$, 5 우 우 (ZMTU), same area, Khasan Distr., near Andreevka, $42^{\circ} 35^{\prime} \mathrm{N}$, 1311'ㄹ [5], 11-15.07.1998, Y.M.; $1 \sigma^{\star}$ (ZMMU), same area, "Kedrovaya Pad" Res., Kedrovka River [8], August 1990, S.G.; $1 \sigma^{\circ}$ (ZMTU), same area, SE part, ca. $25 \mathrm{~km}$ NE of Nakhodka [15], 18.08.1998, Y.M.; 1 \% (ZMMU), Khabarovsk Province, Boitsovo [91], summer 1990, S.G.; $20^{x} 0^{\star}, 1$ o (ISEA), same area, Obluchie [90], 8.06.-20.07.1999, V.D.

\section{Evarcha arcuata (Clerck, 1758)}

Evarcha arcuata: Azheganova \& Stenchenko 1977: 111; Prószynski 1979: 308; Dunin 1984: 132, figs 11-13; Marusik 1988: 1482; Logunov \& Wesolowska 1992: 115-116; Marusik et al. 1992: 151; Marusik et al. 1993: 82; Kim \& Kurenshchikov 1995: 65.

Localities. Magadan Area: Sibit-Tyellakh River basin [123] (Marusik 1988), Talon Town [113], Koni Peninsula [120], Shirokiy [126] (Marusik et al. 1992). - Amur Area: no precise locality (Azheganova \& Stenchenko 1977). Khabarovsk Prov.: Vysokogornyi [104], Akur River [103], Vanino [102], Korfovsky [95] (Dunin 1984), Bolshoi Khekhtsyr Mt. Range [93], Rybachii Isl. [96] (Logunov \& Wesolowska 1992), Komsomol'sk-na-Amure [101], Nikolaevsk-naAmure [109], Slavyanka [100] (Kim \& Kurenshchikov 1995). - Maritime Prov.: "Kedrovaya Pad' “ Res. [8], Ussuriysky (=Suputinsky) Res. [28], Khanka Lake [39], Anisimovka (=Kangauz) [18] (Prószynski 1979), Vladivostok [10], Dushkino [14], Artem [21] (Dunin 1984). - Sakhalin: Kuznetsovo [50] (Dunin, 1984), Aniva [70] (Marusik et al. 1993), - Kurile Islands: Kunashir [71] (Marusik et al. 1993).

Material examined. $1 \%$ (ISEA), Amur Area, near Blagoveshchensk, Verkhneblagoveshchenskoe [76], 14.05.4.08.1999, V.D.; $25 \sigma^{*}, 27$ 우 (ISEA), Maritime Prov., Chemigovka Distr., Dmitrievka [36], 11.07-26.08.1988, A. Borok; 1 우 (ISEA), same area, Gamova Peninsula, Vityaz'
Bay [4], 20-23.05.1994, V.D.; $2 \sigma^{\star} \sigma^{\star}, 4$ 우 (ZMMU), $15 \sigma^{\star} \delta^{\star}$,

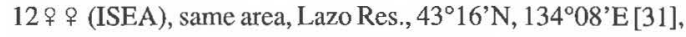
5.06.1975 - 20.06.1984, T.O.; 1 우 (ZMTU), same locality, 69.08.1998, Y.M.; 1 ( (ZMTU), same area, W of Khanka Lake, near Barabash-Levada Vill., 444ㅇ' N, $131^{\circ} 27^{\prime} \mathrm{E}$ [38], 1819.07.1998, Y.M.; $20^{*} \sigma^{*}, 1$ क (ZMTU), same locality, SE shore, $43^{\circ} 39^{\prime} \mathrm{N}, 132^{\circ} 34^{\prime} \mathrm{E}, 15-16.07 .1998$, Y.M.

\section{Evarcha laetabunda (C.L. Koch, 1848)}

Evarcha laetabunda: Dunin 1984: 132, figs 17, 18; Marusik et al. 1993: 82; Logunov 1992: 54; Kim \& Kurenshchikov 1995: 65.

Localities. Khabarovsk Prov.: Sofiysk [108], Komsomol'sk-na-Amure [101] (Dunin 1984), Slavyanka [100], Bolshoi Khekhtsyr Mt. Range [93] (Kim \& Kurenshchikov 1995). - Sakhalin: Beryozovka River [58] (Logunov 1992), Okha [59] (Marusik et al. 1993).

Material examined. 1 ช̛ (ISEA), Khabarovsk Prov., Slavyanka [100], 24.06.1989, D.K. Kurenschikov.

Notes. The record of this species from the Amur Area (Azheganova \& Stenchenko 1977) needs confirmation upon the pertinent material, it may turn out to belong to Evarcha michailovi.

\section{Evarcha proszynskii Marusik \& Logunov, 1988}

Evarcha falcata (misidentification): Kulczynski 1885: 19; Sytshevskaja 1935: 99-100; Azheganova \& Stenchenko 1977: 111; Prószynski 1979: 308; Dunin 1984: 132, figs 14-16; Logunov \& Wesolowska 1992: 116; Marusik et al. 1992: 151; Marusik et al. 1993: 82; Kim \& Kurenshchikov 1995: 65.

Evarcha proszynskii Marusik \& Logunov, 1998 (1997): 101-104, figs 1, 2, 6-8, 14, 19, 20.

Localities. Magadan Area: near Magadan [116] (Marusik \& Logunov 1998). - Amur Area: Khingan Res. [81] (Marusik \& Logunov 1998). Amur Area: no precise locality (Azheganova \& Stenchenko 1977). - Khabarovsk Prov.: Uktur River [105], Vysokogornyi [104], Nizhnetambovskoe [106], Nikolaevask-na-Amure [109] (Dunin 1984: sub Evarcha falcata), Bolshoi Khekhtsyr Mt. Range [93], Ulia River [110] (Marusik et al. 1992: sub Evarcha falcata, Marusik \& Logunov 1998), Boitsovo [91] (Kim \& Kurenshchikov 1995). - Maritime Prov.: Anisimovka (=Kangauz) [18] (Prószynski 1979: sub Evarcha falcata), Lazo Res. [31], Ussuriyski Res. [28] (Marusik 
\& Logunov 1998). - Sakhalin: Ozersky [68], Kostromskoe [53] (Dunin 1984: sub Evarcha falcata), Yuzno-Sakhalinsk [67], Novo-Aleksandrovsk [65], «Lugovoe», «Uspenskoe», Nitui River [61], Pugatchevo [63], Uglegorsk [56], «Utesnoe», Beriozovka River [58], «Slepikovskogo Peninsula» [52] (Marusik et al. 1993: sub Evarcha falcata, Marusik \& Logunov 1998). — Kamchatka: Klyuchevskoe [75] (Kulczynski 1885, Sytshevskaja 1935: both sub Evarcha falcata).

\section{"Harmochirus" latens (Logunov, 1991)}

Harmochirus latens: Logunov \& Wesolowska 1992: 116-117, figs 2, 3; Kim \& Kurenshchikov 1995: 65.

Localities. Amur Area: Khingan Res. [81] (Logunov \& Wesolowska 1992). — Khabarovsk Prov.: Bolshoi Khekhtsyr Mt. Range [93] (Logunov \& Wesolowska 1992). - Maritime Prov.: «Primorskaya station» (Logunov \& Wesolowska 1992).

Material examined. $1 \sigma^{\circ}$ (SMNH), Khabarovsk Province, Amur River Valley, near Slavyanka [100], 14.-18.06.1993, P. Lindskog \& B.Viklund; 10 (ISEA), Amur Area, near Blagoveshchensk [76], August 1996, E.I. Malikova; 1 \%, 1 우 (ISEA), same area, near Blagoveshchensk, Verkhneblagoveshchenskoe [76], 14.05.-4.08.1999, V.D.

\section{'Harmochirus" nigriculus Logunov \& Wesolo- wska, 1992}

Bianor aurocinctus (misidentification): Prószynski 1979: 303-304, figs 15, 16.

Harmochirus nigriculus Logunov \& Wesolowska, 1992: 118-119, figs 4, 5.

Harmochirus nigriculus: Kim \& Kurenshchikov 1995: 65.

Localities. Amur Area: «Kosmodemiyanovka» (Logunov \& Wesolowska 1992). - Khabarovsk Prov.: Bolshoi Khekhtsyr Mt. Range [93], Slavyanka [100] (Logunov \& Wesolowska 1992). Maritime Prov:: Anisimovka (=Kangauz) [18] (Prószynski 1979, Logunov \& Wesolowska 1992).

Notes. We have been unable to re-examine Dunin's (1984) specimens, but very likely his record of Bianor aurocinctus from Vladivostok should be referred to "Harmochirus" nigriculus.

\section{"Harmochirus" pullus (Bösenberg \& Strand, 1906)}

Siler cupreus (misidentification): Sternbergs 1988: 93.

Harmochirus pullus: Logunov \& Wesolowska 1992: 119-120, figs. 6, 7; Kim \& Kurenshchikov 1995: 65.

Localities. Khabarovsk Prov.: Bolshoi Khekhtsyr Mt. Range [93], Rybachii Isl. [96] (Logunov \& Wesolowska 1992, Kim \& Kurenshchikov 1995). - Maritime Prov.: Furugel'ma Isl. [2] (Sternbergs 1988: sub Siler cupreus).

\section{Heliophanus baicalensis Kulczynski, 1895}

Heliophanus baicalensis: Prószynski 1979: 308, figs 98-100; Marusik 1988: 1482; Marusik \& Cutler 1989: 54, figs 4-7; Marusik et al. 1992: 151.

Localities. Magadan Area: Sibit-Tyellakh River basin [123] (Marusik 1988, Marusik \& Cutler 1989), Arman' Pass [115], Gadlya [119], Vetrennyi [124], Ust'-Omtchug [121] (Marusik et al. 1992). - Amur Area: Ulunga River [85] (Prószynski 1979).

\section{Heliophanus camtschadalicus Kulczynski, 1885}

Heliophanus camtschadalicus Kulczynski, 1885: 18, 58 (descr.), tab.11, fig. 35.

Heliophanus ussuricus (misidentification): Marusik 1988: 1482.

Heliophanus camtschadalicus: Wesolowska \& Marusik 1990: 91-92, figs 1-10; Marusik et al. 1992: 151; Marusik et al. 1993: 82; Kim \& Kurenshchikov 1995: 65.

Localities. Magadan Area: Sibit-Tyellakh River basin [123], Dukcha River [116], Detrin River basin [121], Taygonos Peninsula [129], Koni Peninsula [120], Shirokiy [126] (Marusik 1988: sub Heliophanus ussuricus, Wesolowska \& Marusik 1990, Marusik et al. 1992). — Sakhalin: Okha [59] (Marusik et al. 1993). — Kamchatka: Klyuchevskoe [75] (Kulczynski 1885).

Notes. The record from Sakhalin, Uglegorsk [56] (Marusik et al. 1993) turned out to belong to 
Heliophanus ussuricus (Marusik's material reexamined). The record from the Khabarovsk Province, Slavyanka (Kim \& Kurenshchikov 1995) needs confirmation upon the pertinent material, it may also belong to Heliophanus ussuricus.

\section{Heliophanus dubius C.L. Koch, 1848}

Heliophanus dubius: Sternbergs 1988: 93; Marusik 1988: 1482; Logunov \& Wesolowska 1992: 120; Marusik et al. 1992: 151; Marusik et al. 1993: 82; Kim \& Kurenshchikov 1995: 65.

Localities. Magadan Area: Sibit-Tyellakh River basin [123] (Marusik 1988, Marusik et al. 1992). - Khabarovsk Prov.: Bolshoi Khekhtsyr Mt. Range [93] (Logunov \& Wesolowska 1992), Nikolaevsk-na-Amure [109] (Kim \& Kurenshchikov 1995). - Maritime Prov.: Furugel'ma Isl. [2] (Sternbergs 1988). — Sakhalin: Aniva [70], Korsakov [69], Makarov [62] (Marusik et al. 1993).

Material examined. 1 \% (ISEA), Amur Area, near Blagoveshchensk, Verkhneblagoveshchenskoe [76], 14.05.4.08.1999, V.D.

\section{Heliophanus flavipes (Hahn, 1831)}

Heliophanus flavipes: Azheganova \& Stenchenko 1977: 111.

Localities. Amur Area: no precise locality (Azheganova \& Stenchenko 1977).

\section{Heliophanus lineiventris Simon, 1868}

Heliophanus lineiventris: Prószynski 1979: 309; figs 107-109; Dunin 1984: 132; Logunov \& Wesolowska 1992: 120-121; Marusik et al. 1993: 82; Kim \& Kurenshchikov 1995: 65.

Localities. Khabarovsk Prov.: Troitskoe [99], «Malvinskoe» (?) near Okhotsk sea (Prószynski 1979), Khabarovsk [96], Bolon' Lake [98] (Logunov \& Wesolowska 1992), Pashkovo [89], Slavyanka [100] (Kim \& Kurenshchikov 1995). - Maritime Prov.: Anisimovka (=Kangauz) [18], Khanka Lake [39] (Prószynski 1979), Pokrovka [25] (Dunin 1984). - Sakhalin: Kostromskoe [53], Ozersky [68], Aniva [70], Korasakov [69] (Dunin 1984, Marusik et al. 1993). — Kurile Islands: Kunashir [71] (Dunin 1984, Marusik et al., 1993).
Material examined. $10^{*}, 3$ 우 (ISEA), Amur Area, near Blagoveshchensk, 7-8 km NE of Mokhovaya Pad', Peschanoe Lake [76], 4-5.07.1999, V.D.; 1 \% (ISEA), Maritime Prov., ca. $5 \mathrm{~km} \mathrm{~S}$ of Smolyaninovo, Romanovka [19], 1-13.05.1994, V.D.; $110^{\top} 0^{\top}, 6$ 우우 (ISEA), same area, Lazo Res., $43^{\circ} 16^{\prime} \mathrm{N}$, 134 08'E [31], 15.07.1975-20.09.1981, T.0.; 2 ㅇ (ZMTU), same locality, 6-9.08.1998, Y.M.; 1 ㅇ (ZMTU), Maritime Prov., S part, $43^{\circ} 17^{\prime} \mathrm{N}, 134^{\circ} 12^{\prime} \mathrm{E}$, 5.08.1998, Y.M.

\section{Heliophanus patagiatus Thorell, 1875}

Heliophanus patagiatus: Marusik 1988: 1482; Marusik et al. 1992: 151.

Localities. Magadan Area: Sibit-Tyellakh River basin [123], Vetrennyi [124], Ust'-Omtchug [121], the middle reaches of Cholomdzha River [112], Seimtchan Town [128] (Marusik 1988, Marusik et al. 1992).

\section{Heliophanus ussuricus Kulczynski, 1895}

Heliophanus ussuricus Kulczynski, 1895: 51-54, figs 6-9.

Heliophanus ussuricus: Prószynski 1979: 310, 119-129; Dunin 1984: 133-134, figs 22-24; Wesolowska \& Marusik 1990: 95; Logunov \& Wesolowska 1992: 121; Marusik et al. 1993: 82; Kim \& Kurenshchikov 1995: 65.

Localities. Khabarovsk Prov.: Bolshoi Khekhtsyr Mt. Range [93] (Wesolowska \& Marusik 1990, Logunov \& Wesolowska 1992), Pashkovo [89], Komsomol'sk-na-Amure [101], Priamuskaya Station [94], Slavyanka [100] (Kim \& Kurenshchikov 1995). — Maritime Prov.: Kedrovaya Pad' Res. [8], Anisimovka (=Kangauz) [18], Khanka Lake [39], Ulunga River [47] (Prószynski 1979), Dushkino [14], Tikhookeanskiy [12], Kiparisovo [22], Blagodatnyi [45], Vladivostok [10], Artem [21] (Dunin, 1984). Sakhalin: Aniva [70], Alexandrovsk-Sakhalinsky [57], Dolinsk [64], Korsakov [69], Kholmsk [52], Makarov [62], Okha [59], Tomari [54], Uglegorsk [56] (Marusik et al. 1993). — Kurile Islands: Kunashir [71], Iturup [72] (Wesolowska \& Marusik 1990, Marusik et al. 1993).

Material examined. 7 우 (ISEA), (ISEA), Maritime Prov., Chernigovka Distr., Dmitrievka [36], 11.0726.08.1988, A.Borok; 6 우 우 (ISEA), $5 \sigma^{7}$ đo 10 우 (ZMMU), same area, Lazo Res., $43^{\circ} 16^{\prime} \mathrm{N}, 134^{\circ} 08^{\prime} \mathrm{E}$ [31], 26.08 .1977 7.09.1979, T.O.; 5 \& क (ZMTU), Khanka Lake, SE shore, $43^{\circ} 39^{\prime} \mathrm{N}, 132^{\circ} 34^{\prime} \mathrm{E}, 15-16.07 .1998$, Y.M.; 2 \% (ISEA), Kurile Islands, Kunashir Isl., ca. 1.5 km W of South-Kurilsk [71], 1-24.08.1994, K.E.; 1 q (ISEA), Amur Area, Blagovesh- 
chensk Distr., ca. $6 \mathrm{~km} \mathrm{~N}$ of Belogorie [77], 7.07.1994, E.I.Malikova; 1 q (ISEA), same area and distr., Sadovoe [78], 5.07.1994, E.I.Malikova; $7 \sigma^{\circ}, 11 \% \circ$ (ISEA), same area, near Blagoveshchensk, Verkhneblagoveshchenskoe [76], 14.05.-4.08.1999, V.D.; 1 o (ISEA), Sakhalin, Uglegorsk [56], 29-30.07.1992, A.M. Basarukin.

\section{Marpissa milleri (Peckham \& Peckham, 1894)}

Marpissa dybowskii Kulczynski 1895: 63-68, figs 36, 41, 42.

Marpissa dybowskii: Prószynski 1979: 311, figs 156-170; Dunin 1984: 135, figs 36, 37; Logunov \& Wesolowska 1992: 124; Kim \& Kurenshchikov 1995: 65.

Marpissa koreanica: Sternbergs 1988: 93.

Marpissa milleri: Logunov 1999: 37-39, figs 6263.

Localities. Khabarovsk Prov.: confluence of Kiya and Ussuri Rivers [92] (Prószynski 1979), «Digun» (?) (Logunov \& Wesolowska 1992), Pashkovo [89] (Kim \& Kurenshchikov 1995). Maritime Prov.: Kedrovaya Pad' Res. [8], Ussuriysky (=Suputinsky) Res. [28], Vinogradovka [29], Slavyanka [7], Kiparisovo [22], Vladivostok [10] (Prószynski 1979, Dunin 1984, both sub Marpissa dybowskii, Sternbergs 1988: sub Marpissa koreanica), Ryazanovka [6] (Logunov \& Wesolowska 1992), Lazo Res. [31] (Logunov 1999). Kurile Islands: Kunashir [71] (Logunov 1999).

\section{Marpissa pomatia (Walckenaer, 1802)}

Marptusa pomatia: Kulczynski 1895: 63. Marpissa pomatia: Kulczynski 1926: 36; Azheganova \& Stenchenko 1977: 111; Prószynski 1979: 312-313, figs 184-199; Dunin 1984: 135, figs 40, 41; Logunov \& Wesolowska 1992: 127-128; Marusik et al. 1993: 82; Kim \& Kurenshchikov 1995: 65; Logunov 1999: 40-41, figs 1-3, 6, 18, 34, 47, 51, 54-55.

Marpissa radiata (misidentification): Sytshevskaja 1935: 100.

Localities. Kamchatka: Klyuchevskoe [75] (Kulczynski 1926). — Amur Area: no precise locality (Azheganova \& Stenchenko 1977), Selemdzhinsk [84] (Logunov \& Wesolowska 1992), Blagoveshchensk [76] (Logunov 1999). Khabarovsk Prov.: Sofiysk [108] (Prószynski 1979), Tsymmermanovka [107], «Malvinskoe» (?) near Okhotsk sea (Prószynski 1979), Bolshoi
Khekhtsyr Mt. Range [93], «Asyat River» (Logunov \& Wesolowska 1992), Nikolaevsk-naAmure [109], Boitsovo [91], Slavyanka [100] (Kim \& Kurenshchikov 1995). - Maritime Prov.: Kedrovaya Pad' Res. [8], «Khutara Bay», «Den», «Dungari», «Gelga Lake», «Navozovo», «Sakhode», «Tambovskoe», Ulunga River [47], Vinogradovka [29] (Prószynski 1979), Vladivostok [10] (Dunin 1984), Lazo Res. [31], Ussuriysky Res. [28] (Logunov 1999). - Sakhalin: «Shebunino», (Dunin 1984), Aniva [70], Korsakov [69], Makarov [62], Okha [59], Nevel'sk [51] (Marusik et al. 1993). - Kurile Islands: Kunashir [71]: Alekhino, Goryacheye, Tretiyakovo (Dunin 1984), Shikotan [73] (Logunov 1999), Iturup [72] (Marusik et al. 1993).

Material examined. $1 \sigma^{\circ}$ (ISEA), Khabarovsk Prov., Obluchie [90], 8.06.-20.07.1999, V.D.

\section{Marpissa pulla (Karsch, 1879)}

Menemerus pulla: Sternbergs 1988: 93.

Marpissa pulla: Logunov \& Wesolowska 1992: 129; Kim \& Kurenshchikov 1995: 65; Logunov 1999: 42, figs 9, 32, 64-65.

Localities. Khabarovsk Prov.: Bolshoi Khekhtsyr Mt. Range [123], «Asyat River» (Logunov \& Wesolowska 1992, Kim \& Kurenshchikov 1995). — Maritime Prov.: Furugel'ma Isl. [2] (Sternbergs 1988: sub Menemerus pulla), Sadgorod [11] (Logunov \& Wesolowska 1992).

\section{Mendoza canestrinii (Ninni in Canestrini \& Pavesi, 1868)}

Marpissa pichoni: Dunin 1984: 135, figs 42-44. Marpissa obscura (misidentification): Nenilin 1985: 132.

Marpissa magister: Logunov \& Wesolowska 1992: 125-126, figs 12, 13; Marusik \& Logunov 1994: 132, figs 3-5; Kim \& Kurenshchikov 1995: 65.

Mendoza canestrinii: Logunov 1999: 49-51, figs 14-15, 24-25, 29, 37, 39, 44, 78-79, 98-104, 107 $108,114,119,123-124,131-132$.

Localities. Amur Area: Blagoveshchensk [76] (Logunov 1999). - Khabarovsk Prov.: Bolshoi Khekhtsyr Mt. Range [123], «Nizhnespasskoe» (Logunov \& Wesolowska 1992), Pashkovo [89] (Kim \& Kurenshchikov 1995). — Maritime Prov.: 
Tchistovodnoe [16], Pokrovka [25] (Dunin 1984: sub Marpissa pichoni), Khasan [1] (Logunov \& Wesolowska 1992: sub Marpissa magister; Marusik \& Logunov 1994: sub Marpissa magister), Khanka Lake [39] (Logunov 1999).

\section{Mendoza dersuuzalai (Logunov \& Wesolows- ka, 1992)}

Marpissa dersuuzalai Logunov \& Wesolowska, 1992: 121-124, figs 8-10.

Marpissa dersuuzalai: Kim \& Kurenshchikov 1995: 65.

Mendoza dersuuzalai: Logunov 1999: 52, figs 105-106, 11, 121, 125-126,

Localities. Amur Area: Khingansk Res. [81] (Logunov \& Wesolowska 1992). — Khabarovsk Prov.: Bolshoi Khekhtsyr Mt. Range [123], «Asyat River» (Logunov \& Wesolowska 1992, Kim \& Kurenshchikov 1995).

\section{Mendoza elongata (Karsch, 1879)}

Marpissa nobilis (misidentification): Prószynski 1979: 312, figs 171-177; Logunov \& Wesolowska 1992 (in part, female only): 126-127, fig.15. Marpissa elongata: Dunin 1984: 135, figs 38, 39; Sternbergs 1988: 93; Logunov \& Wesolowska 1992: 124-125; Marusik et al. 1993: 82; Kim \& Kurenshchikov 1995: 65.

Mendoza elongata: Logunov 1999: 53, figs 45, 122.

Localities. Amur Area: Khingan Res. [81], Lebedinskoe Lake [80] (Logunov \& Wesolowska 1992: sub Marpissa elongata). — Khabarovsk Prov.: Zelyonyi Is1. [96] (Logunov \& Wesolowska 1992: sub Marpissa elongata), Priamurskya Station [94], Komsomol'sk-na-Amure [101] (Kim \& Kurenshchikov 1995: sub Marpissa elongata). - Maritime Prov.: Khanka Lake [39] (Prószynski 1979: sub Marpissa nobilis), Monakino [24] (Dunin 1984), Ussuriyski Res. [28] (Sternbergs 1988: sub Marpissa elongata), Kamen'-Rybolov (Logunov \& Wesolowska 1992: sub Marpissa elongata), Dmitrievka [36] (Logunov 1999). Kurile Islands: Kunashir [71] (Marusik et al. 1993: sub Marpissa elongata).

\section{Mendoza nobilis (Grube, 1861)}

Attus nobilis Grube, 1861: 28.

Marpissa nobilis: Prószynski 1971: 212-214, figs 16-19; Logunov \& Wesolowska 1992 (in part, male only): 126-127, fig. 14; Kim \& Kurenshchikov 1995: 65.

Marpissa pulchra (misidentification): Logunov \& Wesolowska 1992 (in part, female only): 128-129, fig. 17.

Mendoza nobilis: Logunov 1999: 53-55, figs 1617, 26-27, 38, 46, 113, 120.

Localities. Amur Area: Khingan Res. [81], Arkhara [79] (Logunov \& Wesolowska 1992), Malaya Sezanka [83], Kundur [82] (Logunov 1999). - Khabarovsk Prov.: Zelyonyi Isl. [96] (Logunov \& Wesolowska 1992), Bolshoi Khekhtsyr Mt. Range [93] (Kim \& Kurenshchikov 1995), Obluchie [90] (Logunov 1999). - Maritime Prov.: Ussuri River [26], Kamen'-Rybolov [39] (Grube 1861, Logunov \& Wesolowska 1992), Lazo Res. [31], Khasan Lake [39] (Logunov 1999).

Material examined. $10^{*}$ (ISEA), Amur Area, near Blagoveshchensk, Verkhneblagoveshchenskoe [76], 14.05.4.08.1999, V.D.

\section{Mendoza zebra (Logunov \& Wesolowska, 1992)}

Marpissa zebra Logunov \& Wesolowska, 1992: 129-130, fig. 18.

Marpissa zebra: Kim \& Kurenshchikov 1995: 65. Mendoza zebra: Logunov 1999: 57.

Localities. Amur Area: Khingan Res. [81] (Logunov \& Wesolowska 1992). — Khabarovsk Prov.: Bolshoi Khekhtsyr Mt. Range [93] (Logunov \& Wesolowska 1992, Kim \& Kurenshchikov 1995).

\section{Myrmarachne formicaria (De Geer, 1778)}

Myrmarachne formicaria: Prószynski 1979: 313, figs 219-221; Dunin 1984: 135, figs 45, 46; Logunov \& Wesolowska 1992: 120-124, figs 19-21; Marusik et al. 1993: 82; Kim \& Kurenshchikov 1995: 65.

Myrmarachne japonica: Oliger 1984 (?): 123-126, figs $1,2$. 
Localities. Amur Area: Khingan Res. [81] (Logunov \& Wesolowska 1992). - Khabarovsk Prov.: Bolshoi Khekhtsyr Mt. Range [93] (Logunov \& Wesolowska 1992), Komsomol'sk-naAmure [101] (Kim \& Kurenshchikov 1995). Maritime Prov.: Kedrovka River [8], Vinogradovka [29] (Prószynski 1979), Tikhookeanskiy [12], Vladivostok [10] (Dunin 1984), Ussuriyski Res. [28] (Logunov \& Wesolowska 1992), Lazo Res. [31] (Oliger 1984: sub Myrmarachne japonica). — Sakhalin: «Sernovodsk» (Dunin 1984), Moneron [48] (Marusik et al. 1993). — Kurile Islands: Kunashir [71] (Dunin 1984).

Material examined. $1 \sigma^{*}$ (ISEA), Kurile Islands, Shiko$\tan$ Isl. [73], 1.08.1994, K.Y.Eskov; $20^{\circ} \sigma^{\circ}$ (ISEA), Kurile Islands, Zelyony Isl. [74], 6.08.1994, K.Y.Eskov; 1 \% (ZMMU), Sakhalin Area, Moneron Isl. [48], Kogogerasa bay, date ?, A.M.Basarukin; $40^{\circ} 0^{\star}, 4$ 우 9 (ISEA), Maritime Prov., Lazo Res., $43^{\circ} 16^{\prime} \mathrm{N}, 134^{\circ} 08^{\prime} \mathrm{E}$ [31], 11.05.1975-2.10.1984, T.O.; 1 을 (ZMTU), same locality, 6-8.08.1998, Y.M.; 1 1 ; 3 우우 (ISEA), Amur Area, near Kundur [82], 10.06.1999, V.D.

\section{Myrmarachne lugubris (Kulczynski, 1895)}

Salticus lugubris Kulczynski, 1895: 46-50, figs 1-5.

Myrmarachne lugubris: Prószynski 1979: 313314, figs 222-223; Dunin 1984: 136-137, figs 4749; Logunov \& Wesolowska 1992: 133-135, figs 21-23; Kim \& Kurenshchikov 1995: 65.

Myrmarachne inermichelis (misidentification): Oliger 1984: 126, fig. 3.

Localities. Khabarovsk Prov.: Bolshoi Khekhtsyr Mt. Range [93], Slavyanka [100] (Logunov \& Wesolowska 1992), Komsomol'sk-na-Amure [101] (Kim \& Kurenshchikov 1995). — Maritime Prov.: Khanka Lake [39], Anisimovka (=Kangauz) [18] (Prószynski 1979), Monakino [24], Kiparisovo [22], Blagodatnyi [45], Barabash-Levada [38], Vladivostok [10], Turiy Rog [40], Artem [21] (Dunin 1984, Logunov \& Wesolowska 1992), Lazo Res. [31] (Oliger 1984: sub Myrmarachne inermichelis).

Material examined. $60^{\circ} \sigma^{\star}, 11$ ㅇ 9 (ISE, det. hitherto as M. inermichelis by T.O.), $29 \%$ (ZMMU), Maritime Prov., Lazo Res., $43^{\circ} 16^{\prime} \mathrm{N}, 134^{\circ} 08^{\prime} \mathrm{E}$ [31], 4.08.1975-20.06.1981, T.O.; $10^{\star}, 3$ 우 (ISEA), Amur Area, near Blagoveshchensk, Verkhneblagoveshchenskoe [76], 14.05.-4.08.1999, V.D.

\section{Neon reticulatus (Blackwall, 1853)}

Neon reticulatus: Prószynski 1979: 314, fig. 227;
Marusik et al. 1993: 82.

Localities. Maritime Prov.: Kedrovka River [8] (Prószynski 1979). — Sakhalin: Aniva [70], Dolinsk [64], Kholmsk [52] (Marusik et al. 1993). - Kurile Islands: Iturup [72] (Marusik et al. 1993).

Material examined. 1 (ISEA), Kurile Islands, Iturup Isl., Chapan Peninsula [72], 16.08.1994, K.Y.Eskov; 39 \% (ZMMU), 5 \% (ISEA), Kunashir Isl., ca.1.5 km W of Yuzhno-Kuril'sk [71], 1-24.08.1994, K.E.; 3 우 우 (ISEA), Zelyonyi Isl, Srednee Lake [74], 6.08.1994, K.E.; 1 ㅇ (ZMMU), Maritime Prov., "Peter-the-Great" Bay, Popov Isl. [9], 31.08.1991, K.M.; 2 우 (ZMTU), Vladivostok, Akademgorodok [10],

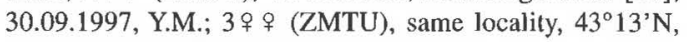
$132^{\circ} 01^{\prime}$ E, 2.10.1997, Y.M.; $1 \%$ (ISEA), same area, «Kedrovaya Pad'» Res. [8], August 1990, A.V.Tanasevitch; 2 \% $\subsetneq$ (ISEA), same area, Lazo Res., Sukhoi Klyuch Stand [31], $43^{\circ} 16^{\prime} \mathrm{N}, 134^{\circ} 08^{\prime} \mathrm{E}, 18.06 .1981$, T.O.; 1 \% (ZMTU), same locality, 6-9.08.1998, Y.M.; 2우우 (ZMMU), same area, Chernigovka Distr., near Merkushevka [35], 20.07.1991, A.V.Tanasevitch; 19 (ZMMU), 2 $\%$ (ISEA), same area, Ussuriysk [26], 19-20.07.1991, S.G.; 2 우 (ZMTU), same area, Pravaya Izvilinka River, $43^{\circ} 55^{\prime} \mathrm{N}, 134^{\circ} 23^{\prime} \mathrm{E}$ [33], 12.08.1998, Y.M.; 1 đ̛ (ISEA), Maritime Prov., Ussuryisk Distr., near Gornotaezhnoe [27], 30.06.1980, V. Belov.

\section{Pellenes (Pelmultus) gobiensis Schenkel, 1936}

Pellenes gobiensis: Logunov \& Marusik 1994: 113.

Localities. Magadan Area: Shirokiy [126] (Logunov \& Marusik 1994).

\section{Pellenes (Pelpaucus) ignifrons (Grube, 1861)}

Pellenes ignifrons: Prószynski 1979: 314; Marusik 1988: 1482; Marusik et al. 1992: 151.

Localities. Magadan Area: Sibit-Tyellakh River basin [123] (Marusik 1988), Kulu [125], Vetrennyi [124], Ust'-Omtchug [121], Seimtchan Town [128], Talaya River mouth [118] (Marusik et al. 1992). - Khabarovsk Prov.: «Chega» (Prószynski 1979).

Material examined. 1 ( 7 (ISEA), Amur Area, ca. $65 \mathrm{~km} \mathrm{~N}$ of Tyndinski [87], 17.06.1997, A. Dudko \& D. Lomakin.

\section{Pellenes (Pelpaucus) limbatus Kulczynski, 1895}

Pellenes limbatus: Marusik 1988: 1482; Logunov 1992: 61, fig. 6.

Localities. Magadan Area: Sibit-Tyellakh River basin [123] (Marusik 1988, Logunov 1992). 


\section{Pellenes (s.str.) sibiricus Logunov \& Marusik, 1994}

Pellenes tripunctatus (misidentification): Kulczynski 1926: 36-37; Sytshevskaja 1935: 100; Marusik 1988: 1482; Marusik et al. 1992: 151. Pellenes sibiricus Logunov \& Marusik, 1994: 108110, figs 6-8.

Localities. Magadan Area: Sibit-Tyellakh River basin [123], Shirokiy [126], Vetrennyi [124] (Marusik 1988: sub Pellenes tripunctatus, Marusik et al. 1992: sub Pellenes tripunctatus, Logunov \& Marusik 1994). - Kamchatka: Klyuchevskoe [75] (Kulczynski 1926: sub Pellenes tripunctatus).

\section{Philaeus chrysops (Poda, 1761)}

Philaeus bicolor: Kulczynski 1895: 73.

Philaeus chrysops: Prószynski 1979: 315, figs 241-254; Dunin 1984: 137, fig. 50.

Localities. Maritime Prov.: Khanka Lake [39], Ussuriysk [26] (Prószynski 1979), Artem [21] (Dunin 1984).

\section{Phintella arenicolor (Grube, 1861)}

Attus arenicolor Grube, 1861: 27.

"Attus" arenicolor: Prószynski 1971: 207, figs $1-6$.

Icius difficilis: Prószynski 1979 (female only): 311, figs 143-144; Dunin 1984 (female only): 134, fig. 33; Sternbergs 1988: 93.

Phintella arenicolor: Logunov \& Wesolowska 1992: 135-137, figs 24-27; Kim \& Kurenshchikov 1995: 65.

Phintella mellotei: Marusik \& Logunov 1994: 135.

Localities. Khabarovsk Prov.: Bolshoi Khekhtsyr Mt. Range [93], Slavyanka [100], Zelyonyi Isl. [96] (Logunov \& Wesolowska 1992, Kim \& Kurenshchikov 1995). — Maritime Prov.: SpasskDal'niy [41], Ussuriysky (=Suputinsky) Res. [28] (Prószynski 1979: sub Icius difficilis, female only), Dushkino [14], Tikhookeanskiy [12] (Dunin 1984: sub Icius difficilis), Kedrovaya Pad' Res. [8], «Primorskaya Station», Bikin River [46], Khorol' [37], Kamen'-Rybolov [39] (Logunov \& Wesolowska 1992), Furugel'ma Isl. [2] (Sternbergs 1988: sub
Icius difficilis).

Material examined. 10 (ISEA), Maritime Prov., Chernigovka Distr., Dmitrievka [36], 11.07-26.08.1988, A. Borok; 1 (ISEA), same area, Khanka Lake, CE shore, $44^{\circ} 39^{\prime} \mathrm{N}$, 1323'ㄹ, 15-16.07.1998, Y.M.

\section{Phintella castriesiana (Grube, 1861)}

Attus castriesiana Grube, 1861: 26.

Maevia castriesiana (?): Kulczynski 1895: 96.

Telamonia castriesiana: Prószynski 1971: 223224.

Icius castriesianus: Prószynski 1979: 310-311, 131-142; Dunin 1984: 134, figs 28-30.

Phintella castriesiana: Logunov \& Wesolowska 1992: 137-138, figs 27-29; Marusik et al. 1993: 82; Kim \& Kurenshchikov 1995: 66.

Localities. Khabarovsk Prov.: Nizhnetambovskoe [106], Nikolevsk-na-Amure [109] (Dunin 1984: sub Icius castriesianus), Bolshoi Khekhtsyr Mt. Range [93], «Kutuzovka» (Logunov \& Wesolowska 1992), Boitsovo [91], «Voronezhsky Hills», Slavyanka [7], «Priamurskaya Station» (Kim \& Kurenshchikov 1995). - Maritime Prov.: Kedrovaya Pad' Res. [8], Kedrovka River [8], Anisimovka (=Kangauz) [18], Khanka Lake [39] (Prószynski 1979: sub Icius castriesianus), Vladivostok [10], Blagodatnyi [45], Kiparisovo [22] (Dunin 1984: sub Icius castriesianus). — Sakhalin: «Shebunino», Kostromskoe [53] (Dunin 1984: sub Icius castriesianus), Aniva [70], Dolinsk [64], Korsakov [69], Nevel'sk [51], Kholmsk [52], Moneron Isl. [48] (Marusik et al. 1993). — Kurile Islands: Kunashir [71] (Dunin 1984: sub Icius castriesianus), Iturup [72] (Marusik et al. 1993).

Material examined. 1 o 1 \% (ISEA), Maritime Prov., Vladivostok [10], 19-28.05.1994, V.D.; 2 우 우 (ISEA), same area, Anuchino Distr., 10-20 km N of Daubekhaza River Valley [23], 3-4.06.1994, V.D.; 3 $\sigma^{\circ}, 8 \% q$ (ISEA), same area, Nadezhdino Distr., ca. $12 \mathrm{~km}$ NW of Vinevitino, Malya El'duga River [23], 8-9.06.1994, V.D.; $2 \sigma^{\star} 0^{*}, 5$ 우 우 (ISEA), same area, ca. $20 \mathrm{~km}$ SE of Ussuriysk, Gomotaezhnoe [27], 1-2.06.1994, V.D.; 1 ๙ $^{\circ}$ (ISEA), same locality, 06.1990, S.G.;

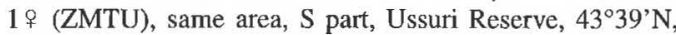

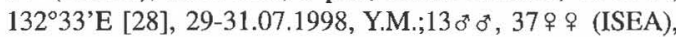

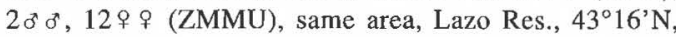
$134^{\circ} 08^{\prime} \mathrm{E}$ [31], 20.05.1975-24.06.1981, T.O.; $10^{\star 7}, 3$ 우 우 (ISEA), same area, Kedrovaya Pad' Res. [8], 5.06.1976, B.P.Zakharov; $1 \sigma^{\ddagger}, 1$ \% (ZMMU), Khabarovsk Province, right bank of Amur River, ca. $20 \mathrm{~km}$ N of Troitskoye, Slavyanka [100], 14-19.06.1990, S.G.; $20^{*} \sigma^{7}, 1$ 우 (ZMMU), Boitsovo [91], summer 1990, S.G. 


\section{Phintella linea (Karsch, 1879)}

Phintella linea: Logunov \& Wesolowska 1992: 138-139, figs 30-31; Marusik et al. 1993: 82; Kim \& Kurenshchikov 1995: 66.

Localities. Khabarovsk Prov.: Bolshoi Khekhtsyr Mt. Range [93] (Logunov \& Wesolowska 1992), «Voronezhsky Hills», Slavyanka [100] (Kim \& Kurenshchikov 1995). — Sakhalin: Aniva [70], Kholmsk [52] (Marusik et al. 1993). Kurile Islands: Kunashir [71] (Marusik et al. 1993).

Material examined. $2 \sigma^{\star}$ đ, $3 \% 9$ (ISEA), Kurile Islands, Kunashir Isl., ca. $1.5 \mathrm{~km} \mathrm{~W}$ of Yuzhno-Kurilsk [71], 124.08.1994, K.E.; $2 \sigma^{\star} \sigma^{\star}, 1+$ (ISEA), Kurile Islands, Zelyony Isl., Srednee Lake [74], 6.08.1994, K.E.

\section{Phintella parva (Wesolowska, 1981)}

Icius difficilis: Prószynski 1979 (male only): 311, figs 145-149.

Icius sp.: Prószynski 1979: 340, figs 154, 155.

Icius parvus: Dunin 1984: 134.

Phintella parva: Logunov \& Wesolowska 1992: 139-141, figs 32-33; Kim \& Kurenshchikov 1995: 66.

Localities. Khabarovsk Prov.: Bolshoi Khekhtsyr Mt. Range [93], «Kutuzovka» (Logunov \& Wesolowska 1992), Boitsovo [91] (Kim \& Kurenshchikov 1995). — Maritime Prov.: Khanka Lake [39] (Prószynski 1979: sub Icius difficilis, male only), Chuguevka [43], Ussuriyski Res. [28] (Logunov \& Wesolowska 1992).

Material examined. $20^{*} 0^{*}, 3$ 우 (ISEA), Maritime Prov., Vladivostok [10], 19-28.05.1994, V.D.; 1 \% (ISEA), same area, ca. $20 \mathrm{~km}$ SE of Ussuryisk, Gornotaezhnoe [27], 1-2.06.1994, V.D.; $20^{\star} \sigma^{x}, 4$ ㅇ + (ISEA), same area, Lazo Res., $43^{\circ} 16^{\prime} \mathrm{N}$, $134^{\circ} 08^{\prime} \mathrm{E}$ [31], 11.06.1975-11.06.1978, T.O.

\section{Phintella popovi (Prószynski, 1979)}

Icius popovi Prószynski, 1979: 311, figs 150-153. Icius abnormis (misidentifications): Prószynski 1979: 310, fig. 130; Dunin 1984: 134, figs 25-27. Icius popovi: Dunin 1984: 134-135, figs 34, 35. Phintella popovi: Logunov \& Wesolowska 1992: 141-143, fig. 34; Kim \& Kurenshchikov 1995: 66.

Localities. Amur Area: Khingan Res. [81], «Nizhnepokrovskoe» (Logunov \& Wesolowska
1992). - Khabarovsk Prov.: Khabarovsk [96], Bolshoi Khekhtsyr Mt. Range [93], Pashkovo [89], Slavyanka [100], Slavyanski Isl. [100] (Logunov \& Wesolowska 1992, Kim \& Kurenshchikov 1995). - Maritime Prov.: Anisimovka (=Kangauz) [18], Vinogradovka [29], «Baraudinsk», Spassk-Dal'niy [41], Tigrovoi [17], «Vyatskoe» (Prószynski 1979: sub Icius popovi and Icius abnormis), Dushkino [14], Kiparisovo [22], Blagodatnyi [45], Vladivostok [10], Artem [21] (Dunin 1984: sub Icius popovi and Icius abnormis), Khorol' [37], Bikin River [46], Kedrovaya Pad' Res. [8], Yakovlevka [42], Kamenushka [8], Furugel'ma Isl. [2] (Logunov \& Wesolowska 1992).

Material examined. 2 ㅇ (ISEA), Amur Area, near Kundur [82], 31.07.1996, A.N.Streltsov \& E.I.Malikova;; 1 \%, 1 ㅇ (ISEA), same area, near Blagoveshchensk, Verkhneblagoveshchenskoe [76], 14.05.-4.08.1999, V.D.; 1 o (ISEA), Maritime Prov., ca. $5 \mathrm{~km} \mathrm{~S}$ of Smolyaninovo, Romanovka [19], 113.05.1994, V.D.; $2 \sigma^{\circ} \sigma^{*}, 4$ 우 (ISEA), 2 우 (ZMMU), same arca, Lazo Rcs., $43^{\circ} 16^{\prime} \mathrm{N}, 134^{\circ} 08^{\prime} \mathrm{E}$ [31], 4.06.19757.09.1979, T.O.; 7 우우 (ZMTU), same locality, 6-9.08.1998, Y.M.; 1 (ZMTU), same area, S part, Ussuri Reserve, $43^{\circ} 39^{\prime} \mathrm{N}, 132^{\circ} 33^{\prime} \mathrm{E}$ [28], 29-31.07.1998, Y.M.

\section{Phlegra fasciata (Hahn, 1826)}

Phlegra fasciata: Prószynski 1979: 315, figs 257 259.

Localities. Maritime Prov.: Anisimovka (= Kangauz) [18] (Prószynski 1979).

Material examined. $20^{\circ} \sigma^{\circ}$ (ISEA), Maritime Prov., Lazo

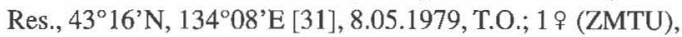
same area, S part, near Anisimovka, $43^{\circ} 10^{\prime} \mathrm{N}, 132^{\circ} 46^{\prime} \mathrm{E}$ [18], 24-28.07.1998, Y.M.; 10 (ISEA), Amur Area, near Kundur [82], 10.06.1999, V.D.

\section{Plexippoides regius Wesolowska, 1981}

Plexippoides regius: Dunin 1984: 137, fig. 51, 52; Kim \& Kurenshchikov 1995: 66.

Localities. Khabarovsk Prov.: Boitsovo [91] (Kim \& Kurenshchikov 1995). - Maritime Prov.: Pokrovka [25] (Dunin 1984).

Material examined. 10 (ISEA), Maritime Prov., ca. 20 km SE of Ussuriysk, Gomotaezhnoe [27], 1-2.06.1994, V.D.; $1 \sigma^{*}$ (ISEA), same locality, 14.06.1989, S.Kurbatov; $40^{*} \sigma^{\circ}, 1 \%$ (ISEA), Lazo Res., $43^{\circ} 16^{\prime} \mathrm{N}, 134^{\circ} 08^{\prime} \mathrm{E}$ [31], 22.06.1981, T.O. 


\section{Pseudeuophrys iwatensis (Bohdanowicz \& Prószynski, 1987)}

Euophrys erratica: Prószynski 1979: 306, figs 6468.

Euophrys iwatensis: Logunov et al. 1993: 106107, fig. 3; Kim \& Kurenshchikov 1995: 65; Marusik et al. 1993: 82.

Pseudeuophrys iwatensis: Logunov 1998b: 119, figs $22,27,28,31,32$.

Localities. Khabarovsk Prov.: Bolshoi Khekhtsyr Mt. Range [93] (Logunov et al. 1993). Maritime Prov.: Kedrovka River [8], Yakovlevka [42] (Prószynski 1979: sub Euophrys erratica), Lazo Res. [31], Sredni Bikin River [46], Furugel'ma Isl. [2] (Logunov 1998b). — Sakhalin: «Tsapko» (Logunov et al. 1993), Dolinsk [64], Makarov [62] (Marusik et al. 1993).

Material examined. 2 o o (ISEA), Maritime Prov., Lazo Res., $43^{\circ} 16^{\prime} \mathrm{N}, 134^{\circ} 08^{\prime} \mathrm{E}$ [31], 6-8.08.1998, Y.M.

\section{Pseudicius chikunii Logunov \& Marusik, 1999}

Pseudicius chikunii Logunov \& Marusik, 1999b: 25-27, figs 5, 10-13, 16.

Localities. Maritime Prov.: Khanka Lake [39] (Logunov \& Marusik 1999).

\section{Pseudicius vulpes (Grube, 1861)}

Pseudicius orientalis: Kulczynski 1895: 59-63, figs 12-14.

Pseudicius vulpes: Prószynski 1979: 316, figs 276-278; Dunin 1984: 137, figs 53-55; Marusik et al. 1993: 82; Logunov \& Wesolowska 1992: 143.

Localities. Khabarovsk Prov.: Slavyanka [100], Khabarovsk [96] (Prószynski 1979), Sofiysk [108] (Dunin 1984), Bolshoi Khekhtsyr Mt. Range [93], Slavyanski Isl. [96] (Logunov \& Wesolowska 1992). - Maritime Prov.: Anisimovka (=Kangauz) [18], Shkotovo [20], «Mikhailovskoe», Ussuriysk [26], Vladivostok [10], Kiparisovo [22], Kedrovaya Pad' Res. [8] (Prószynski 1979, Dunin 1984). — Sakhalin: Kostromskoe [53], Alexandrovsk-Sakhalinsk [57] (Dunin, 1984), Aniva [70], Dolinsk [64], Kholmsk [52] (Marusik et al. 1993). — Kurile Islands: Kunashir [71] (Marusik et al. 1993).

Material examined. 10 (SMNH), Khabarovsk Province,
Amur River Valley, near Slavyanka [100], 13.06.1993, B.Viklund; 19 (ZMTU), Maritime Prov., near Vladivostok (NW part) [10], 21.08.1998, Y.M.; $10 \sigma^{\star} \sigma^{\star}, 3 \%$ (ISEA), $2 \sigma^{\star} \sigma^{\star}$, 1 (ZMMU), same area, Lazo Res. [31], 19.05.19755.07.1982, T.O.; $10^{*}$ (ISEA), same area, Chemigovka Distr., Dmitrievka [36], summer 1991, S.G.; 1 (ISEA), same area, near Vladivostok, "Peter the Great" Bay [9], 6-8.09.1991, K.M.; $1 \sigma^{\star}, 1$ ㅇ (ZMMU), same area, Ussuri Distr., Gornotayozhnoe [27], 9-19.07.1990, S.G.; $5 \sigma^{*} \sigma^{*}, 20$ 우 (ISEA), same area, Khanka Lake (SW shore), Kamen'-Rybolov Cape [39], $44^{\circ} 43^{\prime} \mathrm{N}, 132^{\circ} 05^{\prime} \mathrm{E}, 18.07 .1998$, Y.M.; $1 \sigma^{\star}$ (ZMTU), same

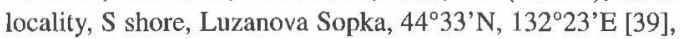
16-17.07.1998, Y.M.; 1 ㅇ (ZMTU), same locality, SE shore, $44^{\circ} 39^{\prime} \mathrm{N}, 132^{\circ} 34^{\prime} \mathrm{E}$ [39], 15-16.07.1998, Y.M.

\section{Rhene atrata (Karsch, 1881)}

Dendryphantes atratus: Prószynski 1979: 304, figs 18-29; Sternbergs 1988: 93.

Rhene atrata: Dunin 1984: 137, figs 56, 57.

Localities. Maritime Prov.: Khanka Lake [39], Kedrovka River [8] (Prószynski 1979), Tchistovodnoe [16], Dushkino [14], Tikhookeanskiy [12], Vladivostok [10], Artem [21] (Dunin 1984), Ussuriyski Res. [28] (Sternbergs 1988: sub Dendryphantes $a$.).

Material examined. 49 ㅇ (ISEA), Maritime Prov., Lazo Res., Kievka [31], $43^{\circ} 16^{\prime} \mathrm{N}, 134^{\circ} 08^{\prime} \mathrm{E}, 15.08 .1975-$ 5.07.1982, T.O.; $2 \sigma^{\circ} \sigma^{7}, 2$ 우 (ZISP), same area, Slavyanka, Tulomu Bay [7], summer 1911, Kuznetsov; 1 ㅇ (ZISP), same area, Kamen'-Rybolov [39], 7.07.1908, A.Chersky; 1 \% (ISEA), same area, SW part, Khasan Distr., near Anisimov$\mathrm{ka}, 42^{\circ} 35^{\prime} \mathrm{N}, 131^{\circ} 13^{\prime} \mathrm{E}$ [18], 11-15.07.1998, Y.M.; 1 웅 (ISEA), same area, S part, Ussuriyski Reserve, $43^{\circ} 39^{\prime} \mathrm{N}$, 132³3’E [28], 29-31.07.1998, Y.M.

\section{Salticus cingulatus (Panzer, 1797)}

Salticus cingulatus: Logunov \& Wesolowska 1992: 143; Kim \& Kurenshchikov 1995: 66.

Localities. Amur Area: Zeya Res. [86] (Logunov \& Wesolowska 1992), Selemdzhinsk [84] (Kim \& Kurenshchikov 1995). - Khabarovsk Prov.: Bolshoi Khekhtsyr Mt. Range [93] (Logunov \& Wesolowska 1992).

\section{Salticus latidentatus Roewer, 1951}

Salticus latidentatus: Logunov \& Marusik 1999b: 27, figs 9, 16.

Localities. Maritime Prov.: Khanka Lake [39] (Logunov \& Marusik 1999b).

Material examined. $1 \sigma^{*}$ (ISEA), Amur Area, near Blagoveshchensk [76], 14.07.1996, A.N.Streltsov. 


\section{Salticus scenicus (Clerck, 1758)}

Salticus scenicus: Logunov \& Marusik 1999b: 2728, figs 14-16.

Localities. Maritime Prov.: Khanka Lake [39] (Logunov \& Marusik 1999b).

\section{Sitticus albolineatus (Kulczynski, 1895)}

Sitticus albolineatus: Prószynski 1979: 316-317, 283-289; Dunin 1984: 137, figs 58-60; Marusik 1988: 1482; Marusik et al. 1992: 151; Logunov 1998a: 81.

Localities. Magadan Area: Sibit-Tyellakh River basin [123] (Marusik 1988), Arman' Pass [115], Vetrennyi [124], Ust'-Omtchug [121], Seimtchan Town [128] (Marusik et al. 1992). - Maritime Prov.: Lazo Res. [31], Anisimovka (=Kangauz) [18], Kedrovaya Pad' Res. [8] (Prószynski 1979; Dunin 1984, Logunov 1998a).

Material examined. 1 \& ('LM'TU), Maritime Prov., S part, $43^{\circ} 17^{\prime} \mathrm{N}, 134^{\circ} 12^{\prime} \mathrm{E}, 5.08 .1998$, Y.M.; $1 \sigma^{\circ}$ (ZMTU), same area, Ussuriyski Res., $43^{\circ} 39^{\prime} \mathrm{N}, 132^{\circ} 33^{\prime} \mathrm{E}$ [28], 29-31.07.1998, Y.M.; $1 \sigma^{\circ}, 5$ 우 (ZMTU), same area, Lazo Res., $43^{\circ} 16^{\prime} \mathrm{N}$, $134^{\circ} 08^{\prime} \mathrm{E}$ [31], 6-9.08.1998, Y.M.

\section{Sitticus cutleri Prószynski, 1980}

Sitticus cutleri: Marusik 1988: 1482; Logunov \& Wesolowska 1992: 143-144; Marusik et al. 1992: 151.

Sitticus catieri (misprint): Kim \& Kurenshchikov 1995: 66.

Localities. Magadan Area: Sibit-Tyellakh River basin [123] (Marusik 1988), Talon Town [113], Kulu [125], Vetrennyi [124], Seimtchan Town [128] (Marusik et al. 1992). - Khabarovsk Prov.: Bolshoi Khekhtsyr Mt. Range [93], Ulia River [110] (Logunov \& Wesolowska 1992, Kim \& Kurenshchikov 1995: sub Sitticus catieri).

\section{Sitticus distinguendus (Simon, 1868)}

Sitticus viduus (misidentification): Prószynski 1979: 317.

Sitticus avocator (misidentification): Logunov 1998a: 81.

Localities. Maritime Prov.: Kedrovaya Pad' Res. [8] (Prószynski 1979: sub Sitticus viduus), Lazo Res. [31] (Logunov 1998a: sub Sitticus avocator).
Material examined. 1 i (ZMTU), Maritime Prov., SE part, ca. $25 \mathrm{~km}$ NE of Nakhodka [15], 18.08.1998, Y.M.; 3 우 우

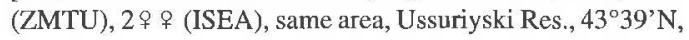
132 $33^{\prime}$ E [31], 29-31.07.1998, Y.M. \& S.K.

\section{Sitticus eskovi Logunov \& Wesolowska, 1995}

Sitticus sp.: Marusik et al. 1992: 151.

Sitticus eskovi Logunov \& Wesolowska, 1995: 164-167, figs 1-8.

Localities. Maritime Prov.: «Sokolovskaya Bay», Furugel'ma Is1. [2] (Logunov \& Wesolowska 1995). — Kurile Islands: Iturup [72], Zelyony [74] (Logunov \& Wesolowska 1995). - Sakhalin: Novo-Aleksandrovsk [65], «Utesnoe», Kril'on Peninsula [49], «Uspenskoe», Moneron Isl. [48] (Marusik et al. 1992: sub Sitticus sp., Logunov \& Wesolowska 1995).

\section{Sitticus fasciger (Simon, 1880)}

Sitticus fasciger: Prószynski 1979: 317; Logunov \& Wesolowska 1992: 144; Kim \& Kurenshchikov 1995: 66; Logunov 1998a: 81.

Localities. Amur Prov.: Blagoveshchensk [76] (Logunov 1998). — Khabarovsk Prov.: Bolshoi Khekhtsyr Mt. Range [93], Kiya River [92] (Logunov \& Wesolowska 1992, Kim \& Kurenshchikov 1995). - Maritime Prov.: Khanka Lake [39], Anisimovka (=Kangauz) [18], Kedrovka River [8] (Prószynski 1979), Gornotaezhnoe [27], Lazo Res. [31] (Logunov 1998a).

\section{Sitticus finschi (L. Koch, 1879)}

Sitticus finschi: Marusik 1988: 1482; Sternbergs 1988: 93; Marusik et al. 1992: 151; Marusik et al. 1993: 82.

Localities. Magadan Area: Sibit-Tyellakh River basin [123] (Marusik 1988), Kulu [125], the middle reaches of Cholomdzha River [112], Seimtchan Town [128] (Marusik et al. 1992). Maritime Prov.: Ussuri Prov. (Sternbergs 1988). - Sakhalin: Okha [59] (Marusik et al. 1993).

Notes. The above record of Sitticus finschi from the Maritime Province (Sternbergs 1988) is in need of a confirmation with the pertinent material. 


\section{Sitticus floricola (C.L. Koch, 1837)}

Sitticus floricola: Prószynski 1979: 317; Logunov \& Wesolowska 1992: 144; Marusik et al. 1992: 151; Kim \& Kurenshchikov 1995: 66.

Localities. Magadan Area: Talon Town [113], Arman' Pass [115] (Marusik et al. 1992). Khabarovsk Prov.: Tsymmermanovka [107] (Prószynski 1979), Bolshoi Khekhtsyr Mt. Range [93] (Logunov \& Wesolowska 1992), Pashkovo [89], Komsomol'sk-na-Amure [101] (Kim \& Kurenshchikov 1995).

Material examined. 1 (SMNH), Khabarovsk Province, Amur River Valley, near Slavyanka [100], 13.06.1993, B. Viklund.

\section{Sitticus lineolatus (Grube, 1861)}

Sitticus lineolatus: Marusik 1988: 1482; Marusik et al. 1992: 151.

Localities. Magadan Area: Sibit-Tyellakh River basin [123] (Marusik 1988), Arman' Pass [115], Koni Peninsula [120], Kontakt Field Station Spring [125], Chaun Bay [130], Amguema River [136], the middle reachers of Chegitun' River [138] (Marusik et al. 1992).

\section{Sitticus penicillatus (Simon, 1875)}

Sitticus penicillatus: Marusik et al. 1992: 151.

Localities. Sakhalin: Kholmsk [52] (Marusik et al. 1992).

\section{Sitticus saxicola (C.L. Koch, 1848)}

Sitticus saxicola: Logunov \& Wesolowska 1995: 173-174, figs 10-16; Marusik et al. 1992: 151.

Localities. Sakhalin: Aniva [70], Dolinsk [64], Kholmsk [52], Korsakovo [69], Okha [59], Tomari [54] (Marusik et al. 1992), Chekhov's Peak [66], «Slepikovsky Cape», Ainskoe Lake [55] (Logunov \& Wesolowska 1995).

\section{Synageles hilarulus (C.L. Koch, 1846)}

Sitticus viduus: Prószynski 1979: 318, fig. 291.

Localities. Maritime Prov.: Anisimovka (=Kangauz) [18] (Prószynski 1979)

\section{Synageles morsei Logunov \& Marusik, 1999}

Synageles morsei Logunov \& Marusik, 1999b: 2829 , figs 6-8, 16.

Localities. Maritime Prov.: Khanka Lake [39] (Logunov \& Marusik 1999b).

\section{Synageles nigriculus Danilov, 1997}

Synageles nigriculus Danilov, 1997: 115, fig. 1. Localities. Amur Area: Khingan Res. [81] (Danilov 1997).

\section{Synageles venator (Lucas, 1836)}

Synageles venator: Prószynski 1979: 318, figs 298-306; Logunov \& Wesolowska 1992: 144; Marusik et al. 1992: 151; Kim \& Kurenshchikov 1995: 66.

Localities. Khabarovsk Prov.: Nikolaevsk-naAmure [109] (Prószynski 1979, Logunov \& Wesolowska 1992), Bolshoi Khekhtsyr Mt. Range [93] (Kim \& Kurenshchikov 1995). - Maritime Prov.: Kedrovka River [8], Ussuriyski Res. [28] (Prószynski 1979, Logunov \& Wesolowska 1992). - Sakhalin: Aniva [70], Dolinsk [64], Kholmsk [52], Korsakov [69], Okha [59] (Marusik et al. 1992).

\section{Synagelides agoriformis Strand in Bösenberg \& Strand, 1906}

Synagelides agoriformis: Prószynski 1979 (females only): 318-319, figs 311-315; Dunin 1984: 139, figs 63, 64; Marusik et al. 1992: 151.

Localities. Maritime Prov.: Kedrovaya Pad' Res. [8], Ussuriyski Res. [28] (Prószynski 1979: females only), Vladivostok [10] (Dunin 1984). Kurile Islands: Kunashir [71] (Marusik et al. 1992).

Material examined. 1 i (ISEA), Maritime Prov., Lazo Res., $43^{\circ} 16^{\prime} \mathrm{N}, 134^{\circ} 08^{\prime} \mathrm{E}$ [31], 18.06.1981, T.O.; $4 \sigma^{\top} \sigma^{*}$ (ISEA), same prov., Khasan Distr., Kedrovaya Pad’ Res., Kedrovka River [8], 31.08.1988, L. A. Nessov.

Notes. See below comments on Synagelides zhilcovae.

\section{Synagelides zhilcovae Prószynski, 1979}

Synagelides agoriformis (misidentification): 
Prószynski 1979 (males only): 318-319, figs 307310.

Synagelides zhilcovae Prószynski, 1979: 319, figs 316-317.

Synagelides zhilcovae: Dunin 1984: 139, fig. 65.

Localities. Maritime Prov.: Kedrovaya Pad' Res. [8] (Prószynski 1979: sub Synagelides zhilcovae and Synagelides agoriformis, males only; Dunin 1984).

Material examined. 10 (ZMMU), Khabarovsk Prov., near Boitsovo [91], summer 1990, S. I. Golovatch; 1 đ (ZMTU), Maritime Prov., near Vladivostok (NW part) [10], 21.08.1998, Y.M.; $10^{*}$ (ISEA), same area, Anuchino Distr., 30.07-6.08.1984, Bakurov; $1 \sigma^{\star}$ (ZMMU), $10^{*}, 3$ 우 우 (ISEA), same area, Lazo Res., $43^{\circ} 16^{\prime} \mathrm{N}, 1^{\circ} 4^{\circ} 08^{\prime} \mathrm{E}$ [31], 07.197723.07.1981, T.O,; 5 ? (ISEA), same area, S part, near Anisi-

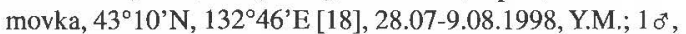
1 ㅇ (ISEA), same area, S part, Ussuriyski Res., 4339' $\mathrm{N}$, $132^{\circ} 33^{\prime} \mathrm{E}$ [28], 29-31.07.1998, Y.M.; $10^{*}, 2$ 우 우 (ISEA), same area, ca. $20 \mathrm{~km}$ SE of Ussuriysk, near Gornotaezhnoe [27], 1-2.06.1994, V.D.; $1 \sigma^{\circ}, 3$ 우 오 (ISEA), same area, Khasan Distr., near Andreevka, $42^{\circ} 35^{\prime} \mathrm{N}, 131^{\circ} 13^{\prime} \mathrm{E}$ [5], 11-15.08.1998, Y.M.; 1 \% (ZMTU), same area, S part, Pravaya Izvilinka River, $43^{\circ} 52^{\prime} \mathrm{N}, 134^{\circ} 17^{\prime} \mathrm{E}$ [33], 2.08.1998, Y.M.; 1 ㅇ (ISEA), same area and distr., Vityaz' Bay, Gamova Peninsula [4], 2023.05.1994, V.D.; 1 \% (ISEA), same area and distr., Popova Isl. [9], 31.07.1978, B. P. Zakharov; 1 i (ISEA), Furugel'ma Isl. [2], 18.07.1975, M. T. Sternbergs; 10 , 1 \% (ISEA), same area and distr., Peter-the-Great Gulf, Nerpich'ya Bay [9], 3.09.1978, B. P. Zakharov; 1 ㅇ (ISEA), Kurile Islands, Kunashir [71], 28.08.1988, A. M. Basarukin; 1 9 (ISEA), same isl., Lesnaya River, $44^{\circ} 01^{\prime}$ N , $145^{\circ} 46^{\prime}$ E [71], 1.09.1995, Y.M.; $1 \sigma^{*}, 2$ 우 (ZMMU), same isl., near Otradnoe [71], 19.09.1987, A. M. Basarukin; $10^{\circ}, 2 \% q$ (ISEA), Kurile Islands, Shikotan Isl., Krabozavodskoe, $43^{\circ} 50^{\prime} \mathrm{N}, 146^{\circ} 46^{\prime} \mathrm{E}$ [73], 10-18.09.1997, Y.M.

Notes. All the males reported and determined hitherto by Prószynski (1979) from the Russian Far East as Synagelides agoriformis in reality belong to Synagelides zhilcovae (Prószynski's specimens re-examined).

\section{Talavera minuta (Banks, 1895)}

Talavera minuta: Marusik 1988: 1482; Marusik et al. 1992: 151; Marusik \& Logunov 1994: 131132 , figs $1,2$.

Localities. Magadan Area: Sibit-Tyellakh River basin (Olen' River) [123] (Marusik 1988, Marusik et al. 1992, Marusik \& Logunov 1994).

\section{Yaginumaella medvedevi Prószynski, 1979}

Yaginumaella medvedevi Prószynski, 1979: 320, figs $318-322$.
Yaginumaella medvedevi: Dunin 1984: 139, figs 66, 67; Logunov \& Wesolowska 1992: 144; Marusik et al. 1992: 151; Kim \& Kurenshchikov 1995: 66.

Localities, Khabarovsk Prov.: Uktur River [105], Sofiysk [108] (Dunin 1984), Bolshoi Khekhtsyr Mt. Range [93] (Logunov \& Wesolowska 1992, Kim \& Kurenshchikov 1995). - Maritime Prov.: Dushkino [14], Domashlino [13], Vladivostok [10], Ussuriyski Res. [28], Kedrovaya Pad' Res. [8], Anisimovka (=Kangauz) [18], Vingoradovka [29], Tigrovoi [17] (Prószynski 1979, Dunin 1984, Logunov \& Wesolowska 1992).

Material examined. 16 , 1 우 (ISEA), Maritime Prov., Vladivostok [10], 19-28.05.1994, V.D.; $10^{n}, 2$ 우 우 (ZMMU), $3 \sigma^{\star} \sigma^{*}, 10$ 우 우 (ISEA), same locality, 6-8.10.1991, K.M.; $10^{*}$, 2 우 우 (ISEA), same area, Nadezhdino Distr., ca. $12 \mathrm{~km} \mathrm{NW}$ of Vinevitino, Malya El'duga River [23], 8-9.06.1994, V.D.; $2 \sigma^{*}, 1$ (ISEA), same area, ca. $20 \mathrm{~km}$ SE of Ussuryisk, Gomotaezhnoe [27], 1-2.06.1994, V.D.; 1 ơ (ZMMU), same locality , 9-19.07.1990, S.G.; 10 (ISEA), same area, Gamova Peninsula, Vityaz' Bay [4], 20-23.05.1994, V.D.; $1 \sigma^{*}$ (ZMTU), same area, near Lazo Town [30], 9.08.1998, Y.M.; $290^{\circ} 0^{\star}, 30$ 우 우 (ISEA), $60^{\star} 0^{\star}, 8$ 우 우 (ZMMU), same area, Lazo Res., $43^{\circ} 16^{\prime} \mathrm{N}, 134^{\circ} 08^{\prime} \mathrm{E}$ [31], 11.05.1975-20.06.1981, T.O.; 2 우 (ZMMU), same area, Ussuriysk [26], 13-20.07.1991, S.G.; 1 \% (ZMMU), same area and distr., Kamenushka [26], 10.06.1989, S.Kurbatov; $10^{*}$ (ZMTU), same area, Pravaya Izvilinka River, $43^{\circ} 55^{\prime} \mathrm{N}, 134^{\circ} 23^{\prime} \mathrm{E}$ [33], 1-2.08.1998, Y.M.; 2 우 우 (ZMMU), Khabarovsk Province, Boitsovo [91], summer 1990, S.G.

Notes. The record from Kurile Islands, Kunashir [71] (Prószynski 1979; Marusik et al. 1992) seems to be based on misidentification. After the first record (Prószynski 1979) we have examined hundred specimens of Yaginumaella from Kunashir, but Y. medvedevi was not found. This species seems to be restricted to the mainland and to be absent from islands.

\section{Yaginumaella striatipes (Grube, 1861)}

Attus striatipes Grube, 1861: 25.

Pellenes striatipes: Prószynski 1971: 219, figs 2829.

Attus striatipes: Azheganova \& Stenchenko 1977: 111.

Yaginumaella striatipes: Prószynski 1979: 320, figs 323-324; Dunin 1984: 139, figs 68-70; Logunov \& Wesolowska 1992: 144; Marusik et al. 1992: 151; Kim \& Kurenshchikov 1995: 66.

Localities. Amur Area: no precise locality (Azheganova \& Stenchenko 1977: sub Attus stri- 
atipes). - Khabarovsk Prov.: Nikolaevsk-naAmure [109] (Grube 1861, Prószynski 1979), Bolshoi Khekhtsyr Mt. Range [93], Slavyanka [100] (Logunov \& Wesolowska 1992), Boitsovo [91], Komsomol'sk-na-Amure [101] (Kim \& Kurenshchikov 1995). - Maritime Prov.: Shkotovo [20] (Prószynski 1979), Vladivostok [10] (Dunin 1984), Chuguevka [43] (Logunov \& Wesolowska 1992). - Sakhalin: «Shebunino» (Dunin 1984), Aniva [70], Alexandrovsk-Sakhalinsky [57], Dolinsk [64], Kholmsk [52], Korsakov [69], Nevel'sk [51], Tomari [54], Moneron Isl. [48] (Marusik et al. 1992). - Kurile Islands: Iturup [72], Kunashir [71] (Marusik et al. 1992).

Acknowledgements. We wish to express our deep gratitude to the colleagues Drs. K.G. Mikhailov (ZMMU), V.I. Ovtsharenko (ZISP), T. Kronestedt (SMNH) and Yu.M. Marusik (Magadan) who loaned or gave us comparative and undetermined salticid material. We thank T. Kronestedt and Yu.M. Marusik also for comments on the manuscript, and V. Rinne (Turku) for help in processing of the text and maps. The work was partially supported by the New Year grant (no. 25) from the Siberian Department of the Russian Academy of Sciences and by the Academy of Finland within the project "Biodiversity and Zoogeography of Arachnids and Insects in North Eurasia".

\section{References}

Azheganova, N. S. \& Stenchenko, T. I. 1977: (To the spider fauna of the Amur Area). - In: Azheganova, N. S. et al. (ed.), Arachno-entomological questions: 106-111. Perm. (In Russian)

Charitonov, D. E. 1932: Katalog der russischen Spinnen AN SSSR, Leningrad, 206 pp.

Danilov, S. N. 1997: New data on the jumping spiders from South Siberia. - Arthropoda Selecta 6(1/2): 113-116.

Dunin, P. M. 1984: (Materials on the spider fauna from the Far East (Arachnida, Aranei). I. The family Salticidae). - In: Ler, P. (ed.), Fauna and ecology of insects in southern Far East: 128-140.Vladivostok. (In Russian)

Grube, A. E. 1861: Beschreibung neuer, von den Herren L. v. Schrenk, Maak, C. v. Dittmar u. a. im Amurlande und in Ostsibirien gesammelter Araneiden. - Bull. Acad. Imp. Sci. S. Pétersburg 4: 161-180.

Ikeda, H. 1996: Japanese salticid spiders of the genera Euophrys C. L. Koch and Talavera Peckham et Peckham (Araneae: Salticidae). — Acta Arachnol. 45(1): 25-41.

Kim, J. P. \& Kurenshchikov, D. K. 1995: Preliminary spider species list (Arachnida, Aranei) of Khabarovsk Territory southem part. - Korean Arachnology 11(1): 55-72.

Kulczynski, W. 1885: Araneae in Camtschadalica a Dra B. Dybowski collectae. - Pam. Wydz. Matem.-przyr. Akad. Krakow 11: 1-60, pls. 9-11.

Kulczynski, VW 1895: Attidae Musei zoologici Varsovien- sis, in Siberia orientali collecti. - Dissert. Akad. Cracoviensis 32: 45-98, pl. 2 .

Kulczynski, W. 1926: Arachnoidea camtschadalica. - Annuaire du Musée Zoologique de l'Acad. Des Sciences l'URSS 27(1): 29-72, pls. 2-3.

Logunov, D. V. 1992: The spider family Salticidae (Araneae) from Tuva. II. An annotated check list of spiders. Arthropoda Selecta 1(2): 47-71.

Logunov, D. V. 1996: Preliminary report on the Euro-Siberian faunal connections of Jumping spiders (Araneae, Salticidae). - Acta Zool. Fennica 201: 71-76.

Logunov, D. V. 1998a: New species and new records of Palaearctic Sitticus (Araneae: Salticidae). - Zoosystematica Rossica 7(1): 77-83.

Logunov, D. V. 1998b: Pseudeuophrys is a valid genus of the jumping spiders (Araneae, Salticidae), - Revue Arachnologique 12(11): 109-128.

Logunov, D.V. 1999: Redefinition of the genera Marpissa C. L. Koch, 1846 and Mendoza Peckham \& Peckham, 1894 in the scope of the Holarctic fauna (Araneae, Salticidae). - Revue Arachnologique 13(3): 25-60.

Logunov, D.V. \& Heciak, S. 1996: Asianellus, a new genus of the subfamily Aelurillinae (Araneae, Salticidae). Entomologica Scandinavica 26: 103-117.

Logunov, D. V. \& Marusik, Yu. M. 1991: (Redescriptions and morphological differences of Bianor aurocinctus (Ohlert) and B. aemulus (Gertsch) (Aranei, Salticidae)). - Siberian Biological Journal 2: 39-47. (In Russian)

Logunov, D. V. \& Marusik, Yu. M. 1994: New data on the jumping spiders of the Palaearctic fauna (Aranei Salticidae). - Arthropoda Selecta 3(1/2): 101-115.

Logunov, D. V. \& Marusik, Yu. M. 1999a: A brief review of the genus Chalcoscirtus Bertkau, 1880 in the fauna of Central Asia and the Caucasus (Aranei; Salticidae). Arthropoda Selecta 7(3): 205-226.

Logunov, D. V. \& Marusik, Yu. M. 1999b: New species and new records of the jumping spiedrs from the Russian Far East (Arancae, Salticidae). - Acta Arachnol. 48: 2329.

Logunov, D. V. \& Rakov, S. Yu. 1998: Miscellaneous notes on Middle Asian jumping spiders (Aranei: Salticidae). - Arthropoda Selecta 7(2): 117-144.

Logunov, D. V. \& Wesolowska, W. 1992: The jumping spiders (Araneae, Salticidae) of Khabarovsk Province (Russian Far East). - Ann. Zool. Fennici 29: 113-146.

Logunov, D. V. \& Wesolowska, W. 1995: New data on some poorly known Palaearctic species of Sitticus (Araneae: Salticidae). - Genus 6(2): 163-175.

Logunov, D. V., Cutler, B. \& Marusik, Y. M. 1993: A review of the genus Euophrys C.L. Koch in Siberia and the Russian Far East (Araneae: Salticidae). - Ann. Zool. Fennici 30: 101-124.

Marusik, Yu. M. 1988: New spider species (Aranei) from the upper Kolyma. —Zool. Zh. 67(10): 1469-1482. (In Russian with English abstract)

Marusik, Yu. M. 1990: The spider genus Chalcoscirtus in the fauna of the USSR. Communication 1. - Zool. Zh. 69(6): 45-56. (In Russian with English abstract)

Marusik, Yu. M. 1991. The spider genus Chalcoscirtus in the fauna of the USSR. Communication 2. - Zool. Zh. 70(1): 19-31. (In Russian with English abstract)

Marusik, Yu. M. \& Cutler, B. 1989: Description of the males 
of Dendryphantes czekanowskii Prószynski and Heliophanus baicalensis Kulczynski (Araneae, Salticidae). - Acta Arachnol. 37: 51-55.

Marusik, Yu. M. \& Logunov, D. V. 1994 (1991): (Poorly known spider species from the families Salticidae and Thomisidae (Aranei) from the Far East of the USSR). Entomol. Studies in North-East USSR 2: 131-140. Vladivostok. (In Russian)

Marusik, Yu. M. \& Logunov, D. V. 1998 (1997): Taxonomic notes on the Evarcha falcata complex (Aranei Salticidae). - Arthropoda Selecta 6(3/4): 95-104.

Marusik, Yu. M., Eskov, K. Yu. \& Kim, J. P. 1992: A checklist of spiders (Aranei) of Northeast Asia. - Korean Arachnology 8(1/2): 129-158.

Marusik, Yu. M., Eskov, K. Yu., Logunov, D. V. \& Basarukin, A. M. 1993: A check-list of spiders (Arachnida Aranei) from Sakahlin and Kurile Islands. - Arthropoda Selecta 1(4): 73-85.

Matsuda, M. 1991: Euophrys erratica (Walckenaer, 1825) and Euophrys iwatensis Bohdanowicz et Prószynski, 1987 (Araneae: Salticidae) found in Hokkaido, Japan. - Bull. Higashi Taisetsu Mus. Nat. Hist. 13: 63-68.

Nenilin, A.B. 1985: (Materials on the fauna of the spiders family Salticidae of the USSR. II. Results of the study in the USSR). - In: Ovtsharenko, V.I. (ed.), Fauna and ecology of spiders of USSR., Trudy Zool. inst. AN SSSR 139: 129-134. (In Russian)

Oliger, T. I. 1984: (Materials on the spider fauna of the Lazo Reservation). - In: Utotchkin, A. (ed.), Fauna and ecology of arachnids: 120-127. Perm. (In Russian)

Peelle, M. L. \& Saito, S. 1933: Spiders from the Southern Kurile Islands. II. Araneidae from Shikotan. - J. Fac. Sci. Hokkaido Imp. Ser. 6 2(3): 109-123.

Prószynski, J. 1971: Redescriptions of A. E. Grube's East
Siberian species of Salticidae (Aranei) in the collection of the Wroclaw Zoological Museum. - Ann. Zool. PAN 28(11): 205-226.

Prószynski, J. 1973: Systematic studies on East Palaearctic Salticidae, II. Redescriptions of Japanese Salticidae of the Zoological Museum in Berlin. - Ann. Zool. PAN 30(5): 97-128.

Prószynski, J. 1979: Systematic studies on East Palaearctic Salticidae. III. Remarks on Salticidae of the USSR. Ann. Zool. PAN 34(11): 299-369.

Prószynski, J. 1990: Catalogue of Salticidae (Araneae). Synthesis of quotations in the world literature since 1940 , with basic taxonomic data since 1758. - Rozprawa Naukowa, WSRP, Siedlce: 336 pp. (http://spiders.arizona edu/proszynski/proszynski.html).

Schenkel, E. 1930: Die Araneiden der schwedischen Kamtchatka-Expedition 1920-1922. - Arkiv för Zoologi 21A(15): 1-33.

Sternbergs, M. T. 1988: (Materials on the spider fauna of the Primorie). - In: Utotchkin, A. (ed.), Fauna and ecology of arachnids: 92-97. Perm. (In Russian)

Sytshevskaja, V. I. 1935: Etude sur les Araignées de la Kamtchatka. - Folia zool. hydrobiol. 8(1): 80-103.

Song, D. X. 1987: Spiders from agricultural regions of China (Arachnida: Araneae). - Agricultural Publishing House, Bejing, 376 pp. (In Chinese)

Yaginuma, T. 1986: Spiders of Japan in color. New Edition. - Hoikusha Publishing Co., Ltd., Osaka, 305 pp. (In Japanese)

Wesolowska, W. \& Marusik, Yu. M. 1990: Notes on Heliophanus camtschadalicus Kulczynski, 1885 (Aranei, Salticidae) and the related species. - Korean Arachnology 6(1): 91-100. 